\title{
Cosmic Time Transformations in Cosmological Relativity
}

\author{
Firmin J. Oliveira \\ East Asian Observatory, Hilo, HI, USA \\ Email: firmjay@hotmail.com
}

Received 10 November 2015; accepted 16 April 2016; published 19 April 2016

Copyright (C) 2016 by authors and Scientific Research Publishing Inc.

This work is licensed under the Creative Commons Attribution International License (CC BY).

http://creativecommons.org/licenses/by/4.0/

(c) (i) Open Access

\section{Abstract}

The relativity of cosmic time is developed within the framework of Cosmological Relativity in five dimensions of space, time and velocity. A general linearized metric element is defined to have the form $\mathrm{ds} s^{2}=(1+\phi) c^{2} \mathrm{~d} t^{2}-\mathrm{d} r^{2}+(1+\psi) \tau^{2} \mathrm{~d} v^{2}$, where the coordinates are time $t$, radial distance $r=\sqrt{x^{2}+y^{2}+z^{2}}$ for spatials $x, y$ and $z$, and velocity $v$, with $c$ the speed of light in vacuum and $\tau$ the Hubble-Carmeli time constant. The metric is accurate to first order in $t / \tau$ and $v / c$. The fields $\phi$ and $\psi$ are general functions of the coordinates. By showing that $\phi=\psi$, a metric of the form $\mathrm{d} s^{2}=c^{2} \mathrm{~d} t^{2}-\mathrm{d} r^{2}+\tau^{2} \mathrm{~d} v^{2}$ is obtained from the general metric, implying that the universe is flat. For cosmological redshift $z$, the luminosity distance relation $D_{L}(z, t)=r(1+z) / \sqrt{1-t^{2} / \tau^{2}}$ is used to fit combined distance moduli from Type 1a supernovae up to $z<1.5$ and Gamma-Ray Bursts up to $\mathrm{z}<7$, from which a value of $\Omega_{M}=0.800 \pm 0.080$ is obtained for the matter density parameter at the present epoch. Assuming a baryon density of $\Omega_{B}=0.038 \pm 0.004$, a rest mass energy of $(9.79 \pm 0.47) \mathrm{GeV}$ is predicted for the anti-baryonic $\bar{Y}$ and the $\Phi^{*}$ particles which decay from a hypothetical $\bar{X}_{1}$ particle. The cosmic aging function $g_{1}(z, t)=(1+z)\left(1-t^{2} / \tau^{2}\right)$ makes good fits to light curve data from two reports of Type 1a supernovae and in fitting to simulated quasar like light curve power spectra separated by redshift $\Delta z \approx 1$. We determine the multipole of the first acoustic peak of the Cosmic Microwave Background radiation anisotropy to be $I \approx 224 \pm 5$ and a sound horizon of $\theta_{\text {sh } 0} \approx(0.805 \pm 0.020)^{\circ}$ on today's sky.

\section{Keywords}

Flat Space, Cosmic Time, Time Dilation, Dark Matter 


\section{Introduction}

It has recently been reported [1] on the apparent null effect of cosmic time dilation upon light curve power spectra measurements of some 800 low and high redshift quasars (QSO) monitored for 28 years. This appears to contradict two Supernovae Type Ia (SNe-Ia) light curve evolution studies [2] [3] which show the effect of broadening of the power spectra time series consistent with a cosmic time dilation of $(1+z)$ for redshift $z$. In this paper, we show that both the QSO and SNe-Ia results are compatible if account is made for the relativity of cosmic time as developed in the theory of Cosmological Special Relativity (CSR) [4]. We also apply these concepts to fitting the combination of high redshift SNe-Ia distance data [5] and Gamma-Ray Bursts (GRB) data [6].

The Cosmological Relativity of Carmeli [7], the general and special theories, is a five dimensional brane world model based on time $t$, space $x, y, z$ and velocity $v$. It makes only one new assumption, that of the maximum value of cosmic time $\tau$ which is the Hubble-Carmeli time constant. In the same way that the constant speed of light $c$ constrains the observations in space and time, so too the constant of cosmic time $\tau$ constrains the observations of space and velocity. The familiar Lorentz transformations of Einstein Special Relativity (SR) between observers moving at constant relative velocity $v$ carry over into Cosmological Special Relativity (CSR) between observers separated by relative cosmic time $t$. And just as in SR, there is the special way that velocities add together which reduces to the Galilean form $v_{1}+v_{2}$ at low velocities with respect to the speed of light, in CSR cosmic times add in an analogous way which has the form $t_{1}+t_{2}$ for low values with respect to cosmic time $\tau$ but is modified for larger cosmic times.

In this paper, we derive only the minimum CSR framework we require for the development of cosmic time transformation effects and the luminosity distance relation. We show that for weak fields $\phi$ and $\psi$ (to be defined below), the universe has a flat, Euclidean geometry. And, due to the additive properties of cosmic time in CSR, this gives us a unique form for the luminosity distance relation. We model cosmic time aging effects in light curve data from SNe-Ia. In comparison to the standard Friedmann-Lemaître-Robertson-Walker (FLRW) model, the CGR luminosity distance relation performs quite well in fits to SNe-Ia and GRB distance data, although it requires more dark matter in the mass density. As a consequence, larger rest masses are predicted for the hypothetical $\bar{X}_{1}$ particle [8] decay products $\bar{Y}$ and $\Phi^{*}$. We also derive a relation for the first acoustic peak of the Cosmic Microwave Background (CMB) anisotropy.

\section{The Universe}

The five dimensional Cosmological General Relativity of Carmeli [7] is approximated by the linearized metric element,

$$
\mathrm{d} s^{2}=(1+\phi) c^{2} \mathrm{~d} t^{2}-\mathrm{d} r^{2}+(1+\psi) \tau^{2} \mathrm{~d} v^{2}
$$

where

$$
\mathrm{d} r^{2}=\mathrm{d} x^{2}+\mathrm{d} y^{2}+\mathrm{d} z^{2} .
$$

The coordinates are time $t$, spatials $x, y$ and $z$ and velocity $v$, with $c$ the speed of light in vacuum and $\tau$ the Hubble-Carmeli time constant. The linearized model is accurate to first order in $v / c \ll 1$ and $t / \tau \ll 1$. The parameter $h=1 / \tau$ is the Hubble constant at zero distance and no gravity and $h \approx H_{0}$ where $H_{0}$ is the Hubble constant. The fields $\phi$ and $\psi$ are general functions of the coordinates. The CGR standard value for $h$ is (to three digits) [9],

$$
h=72.2 \pm 0.84 \mathrm{~km} \cdot \mathrm{s}^{-1} \cdot \mathrm{Mpc}^{-1} .
$$

This gives

$$
\tau=(4.28 \pm 0.15) \times 10^{17} \mathrm{~s}=13.6 \pm 0.48 \mathrm{Gyr} .
$$

In Cosmological Relativity the time coordinate is measured backwards from the present at $t=0$ to the big bang at $t=\tau$. Just as the speed of light $c$ is the maximum observable speed, the time $\tau$ is the maximum observable cosmic time. The gravitational fields are specified by the functions $\phi$ and $\psi$. The expansion of the universe occurs when 


$$
\mathrm{d} s=0 \text {, }
$$

and it is observed at a specific cosmic time $t$, so that

$$
\mathrm{d} t=0 \text {. }
$$

Applying (5) and (6) to (1) yields for the expanding universe

$$
-\mathrm{d} r^{2}+(1+\psi) \tau^{2} \mathrm{~d} v^{2}=0,
$$

which reduces to

$$
\left(\frac{\mathrm{d} r}{\tau \mathrm{d} v}\right)^{2}=1+\psi
$$

The metric (1) defines the Einstein field equations in five dimensions [7] (Sect. 7.3)

$$
R_{\mu}^{v}-\frac{1}{2} \delta_{\mu}^{v} R=\kappa T_{\mu}^{v},
$$

where $R_{\mu}^{v}$ is the mixed Ricci tensor [7] (Appendix A), $R$ is the Ricci scalar and $T_{\mu}^{v}=\rho_{\text {eff }} u_{\mu} u^{v}$ is the mixed energy-momentum tensor, where $\rho_{\text {eff }}$ is the effective mass density and $u_{\mu}=u^{\mu}=(1,0,0,0,1)$ is the velocity vector. The indices $v, \mu=0,1,2,3,4$ for the five dimensions of $x^{0}=c t, x^{1}=x, x^{2}=y, x^{3}=z, x^{4}=\tau v$. The Kronecker delta $\delta_{\mu}^{v}=1$ for $\mu=v$, and $\delta_{\mu}^{v}=0$ for $\mu \neq v$. The Carmeli gravitation constant $\kappa=8 \pi G / c^{2} \tau^{2}$ where $G$ is Newton's gravitation constant. The 0,0 component of (9) gives us the equation

$$
R_{0}^{0}-\frac{1}{2} R=\kappa \tau^{2} \rho_{e f f},
$$

where

$$
\begin{gathered}
R_{0}^{0}-\frac{1}{2} R=\frac{1}{2}\left(\nabla^{2} \phi-\phi_{, 44}-\psi_{, 00}\right)-\frac{1}{2}\left(\nabla^{2} \phi+\nabla^{2} \psi-\phi_{, 44}-\psi_{, 00}\right), \\
=-\frac{1}{2} \nabla^{2} \psi,
\end{gathered}
$$

where $\psi_{, 00}=\partial^{2} \psi / \partial t^{2}$ and $\phi_{, 44}=\partial^{2} \phi / \partial v^{2}$. From (10) and (12) we get the Poisson equation for cosmology, in the space-velocity domain,

$$
\nabla^{2} \psi=-2 \kappa \tau^{2} \rho_{e f f}
$$

The effective mass density is defined by

$$
\rho_{\text {eff }}=\rho-\rho_{c},
$$

where $\rho$ is the mass density and $\rho_{c}$ is the critical mass density. Under the assumption that the mass is uniformly distributed, the mass density $\rho$ is independent of the spatial coordinate $r$, but can depend on time $t$ and velocity $v$. The critical mass density $\rho_{c}$ is a constant defined by

$$
\rho_{c}=3 / 8 \pi G \tau^{2} .
$$

It is useful to express the effective mass density as a parameter in terms of the critical mass density. Dividing by $\rho_{c}$ we have

$$
\Omega_{\text {eff }}=\frac{\rho_{\text {eff }}}{\rho_{c}}=\Omega-1
$$

where $\Omega=\rho / \rho_{c}$ is the mass density parameter. For a spatially uniform mass distribution, $\rho$ is independent 
of $r$, the solution to (13) then takes the form ([7], Sect.7.3.2),

$$
\psi=-\frac{\Omega_{e f f} r^{2}}{c^{2} \tau^{2}}-\frac{2 G M}{c^{2} r},
$$

where $M$ is an optional point mass centered at the origin of $r$. Assuming no central mass, we set $M=0$. Then putting the expression for $\psi$ from (17) into (8) and simplifying we get

$$
\frac{\mathrm{d} r}{\mathrm{~d} v}=\tau \sqrt{1-\Omega_{e f f} r^{2} / c^{2} \tau^{2}}
$$

\section{General Solution in Space-Velocity}

The integration of (18) over $r$ and $v$ in the space-velocity domain is carried out at a specific time $t$. We assume that the mass density $\rho$ is a function of cosmic time only, so that $\Omega_{\text {eff }}$ will be constant throughout the integration. Substitute $\Omega-1=\Omega_{\text {eff }}$ by (16) and put (18) into the integral form

$$
\int_{0}^{r} \frac{\mathrm{d} r^{\prime}}{\sqrt{1+(1-\Omega) r^{\prime 2} / c^{2} \tau^{2}}}=\int_{0}^{v} \tau \mathrm{d} v^{\prime} .
$$

Integrating (19) and solving for $r$ in terms of $v$ we obtain the general solutions

$$
\begin{gathered}
r=\frac{c \tau}{\sqrt{1-\Omega}} \sinh \left(\frac{v}{c} \sqrt{1-\Omega}\right), \text { for } \Omega<1, \\
r=\frac{c \tau}{\sqrt{\Omega-1}} \sin \left(\frac{v}{c} \sqrt{\Omega-1}\right), \text { for } \Omega>1 \text { and } \\
r=\tau v, \text { for } \Omega=1 .
\end{gathered}
$$

By use of the identities $\sinh (i x)=i \sin (x)$ and $\sin (i x)=i \sinh (x)$, where $x$ is real and $i=\sqrt{-1}$, we write the general solution as

$$
r=\frac{c \tau}{\sqrt{1-\Omega}} \sinh \left(\frac{v}{c} \sqrt{1-\Omega}\right) \text {, for } 0 \leq \Omega
$$

\section{Flat Space Metric}

Equation (17) gave the solution for the field $\psi$. Now consider the solution for the field $\phi$ in (1). The 4,4 component of (9) gives us the equation [7]

$$
R_{4}^{4}-\frac{1}{2} R=\kappa \tau^{2} \rho_{\text {eff }}
$$

where

$$
\begin{gathered}
R_{4}^{4}-\frac{1}{2} R=\frac{1}{2}\left(\nabla^{2} \psi-\phi_{, 44}-\psi_{, 00}\right)-\frac{1}{2}\left(\nabla^{2} \phi+\nabla^{2} \psi-\phi_{, 44}-\psi_{, 00}\right), \\
=-\frac{1}{2} \nabla^{2} \phi,
\end{gathered}
$$

where $\psi_{, 00}=\partial^{2} \psi / \partial t^{2}$ and $\phi_{, 44}=\partial^{2} \phi / \partial v^{2}$. Similar to the case for obtaining $\psi$, we solve (26) to obtain

$$
\phi=-\frac{\Omega_{e f f} r^{2}}{c^{2} \tau^{2}}-\frac{2 G M}{c^{2} r},
$$

where $M$ is an optional point mass centered at the origin of $r$. We see by (17) and (27) that in fact,

$$
\phi(r)=\psi(r),
$$

and furthermore, that the constants $c$ and $\tau$ are part of the (cosmological) first term while the constants $c$ and 
$G$ are part of the (Newtonian) second term. Assuming no central mass $(M=0)$, for the case where the mass density becomes equal to the critical density, $\rho \rightarrow \rho_{c}$, from (16), the effective mass density parameter $\Omega_{\text {eff }}=\Omega-1 \rightarrow 0$ and the universe becomes Euclidean.

On the other hand, more generally, we can derive a flatspace metric. Given (28), if we divide (1) by $1+\phi=1+\psi$, we can write

$$
\mathrm{d} \bar{s}^{2}=c^{2} \mathrm{~d} t^{2}-\mathrm{d} \bar{r}^{2}+\tau^{2} \mathrm{~d} v^{2}
$$

where

$$
\bar{s}=\int \frac{\mathrm{d} s}{\sqrt{1+\phi}},
$$

and

$$
\bar{r}=\int \frac{\mathrm{d} r}{\sqrt{1+\phi}},
$$

with the condition that

$$
-1<\phi \text {. }
$$

This implies that for weak fields $\phi$ and $\psi$ we can express the cosmology of the expanding universe in terms of a flat space Euclidean geometry, with a metric of the form $\mathrm{d} s^{2}=c^{2} \mathrm{~d} t^{2}-\mathrm{d} r^{2}+\tau^{2} \mathrm{~d} v^{2}$. In the next section we derive this flat space special theory for cosmology.

\section{The Cosmological Special Relativistic Transformation}

By (29), with notation $\bar{r} \rightarrow r$, we have the metric

$$
\mathrm{d} s^{2}=c^{2} \mathrm{~d} t^{2}-\mathrm{d} r^{2}+\tau^{2} \mathrm{~d} v^{2},
$$

where $r$ is given by (31). The expansion of the universe occurs when $\mathrm{d} s=0$ and observations are made at a particular instant of time $t$ so that $\mathrm{d} t=0$. Then for the expansion, (33) gives us

$$
\mathrm{d} r=\tau \mathrm{d} v,
$$

which, upon integration gives the Hubble law

$$
v=r / \tau=h r
$$

where $h=1 / \tau$.

In the observable universe there are two classes of objects, those that are bounded by the gravitation of their combined masses and those that are observed to be moving away from one another in the Hubble flow. In other words, if we lump all nearby neighboring galaxies into a super galaxy mass point, then the universe would consist of only super galaxy mass points flying apart in the Hubble flow. Cosmological Special Relativity (CSR) describes these super galactic objects in the universe. However, unlike SR which can have real observers in reference frames which move relatively at less than light speed, in CSR, all galaxies are in the Hubble flow and expand at the Hubble rate $h$. That is, there are no objects not in the Hubble flow from which to set up a frame of reference and compare observations. Consequently, for CSR we define hypothetical observers in their frames which move relatively at a rate $1 / t>h$. We derive the transformation of coordinates between these hypothetical frames. However, the coordinates of real galaxies are included in the transformation.

In CSR the age of the universe is $\tau$, the Hubble-Carmeli time constant, which is assumed to be the same for all cosmic time relative inertial observers and is the maximum cosmic time (just as in SR the speed of light $c$ is the maximum velocity and is constant for all velocity relative inertial observers.) Assume that there are hypothetical observers in reference frames $K$ and $K^{\prime}$ separated by a fixed cosmic time $t<\tau$. Each frame is assumed to be unaccelerated (inertial) with respect to cosmic time. Transformations are made between $K$ describing an object $O$ with "4-vector" coordinates $(\tau v, x, y, z)$ and $K^{\prime}$ describing the same object $O$ with 4- 
vector coordinates $\left(\tau v^{\prime}, x^{\prime}, y^{\prime}, z^{\prime}\right)$. The magnitude of each 4-vector is defined by

$$
\begin{gathered}
S^{2}=\tau^{2} v^{2}-x^{2}-y^{2}-z^{2}=\tau^{2} v^{2}-r^{2}, \\
S^{\prime 2}=\tau^{2} v^{\prime 2}-x^{\prime 2}-y^{\prime 2}-z^{\prime 2}=\tau^{2} v^{\prime 2}-r^{\prime 2},
\end{gathered}
$$

with the invariant condition

$$
S^{2}=S^{\prime 2}
$$

where $r=\sqrt{x^{2}+y^{2}+z^{2}}$ and $r^{\prime}=\sqrt{x^{\prime 2}+y^{\prime 2}+z^{\prime 2}}$. For the case that object $O$ is a galaxy in the expansion, the invariants $S^{2}=S^{\prime 2}=0$. We refer to $S$ and $S^{\prime}$ as 4-vectors even though there are only 2 components, since the 3 spatial components $(x, y, z)$ are condensed into $r=\sqrt{x^{2}+y^{2}+z^{2}}$.

To obtain the cosmological transformation ([7], Sect. 2.2), analogous with the Lorentz transformation, assume that a linear transformation exists between the coordinates of hypothetical frames $K$ and $K^{\prime}$. In frame $K$ define the space-velocity 4-vector $S=(\tau v, r)$ with magnitude

$$
S^{2}=\tau^{2} v^{2}-r^{2} \geq 0
$$

Similarly, in frame $K^{\prime}$ define the space-velocity 4-vector $S^{\prime}=\left(\tau v^{\prime}, r^{\prime}\right)$ with magnitude

$$
S^{\prime 2}=\tau^{2} v^{\prime 2}-r^{\prime 2} \geq 0
$$

By the requirement that the magnitude of a 4 -vector is invariant under transformations between reference frames then

$$
S^{2}=S^{\prime 2} \text {. }
$$

Clearly, by (39) and (40), the 4-vectors $S$ and $S^{\prime}$ describe coordinates which can be either in the Hubble flow, when $S^{2}=S^{\prime 2}=0$, or not in the Hubble flow, when $S^{2}=S^{\prime 2}>0$. In order to obtain the transformation equations between the 4-vectors it is required that the reference frames $K$ and $K^{\prime}$ be separated by a fixed cosmic time of $t<\tau$.

Then, for constants $\cosh (\sigma)$ and $\sinh (\sigma)$, where $\sigma$ is a constant hyperbolic angle, define $r^{\prime}$ and $v^{\prime}$ such that

$$
r^{\prime}=r \cosh (\sigma)-\tau \nu \sinh (\sigma)
$$

and

$$
\tau v^{\prime}=\tau \nu \cosh (\sigma)-r \sinh (\sigma) .
$$

To solve for the angle $\sigma$ use the boundary condition $r^{\prime}=0$ which represents the origin of frame $K^{\prime}$. At $r^{\prime}=0 \quad$ (42) yields,

$$
\tanh (\sigma)=\frac{\sinh (\sigma)}{\cosh (\sigma)}=\frac{r}{\tau \nu}=\frac{t}{\tau}
$$

where

$$
t=\frac{r}{v}
$$

is the fixed cosmic time separating the origins of frame $K^{\prime}$ relative to $K$. From (44), for

$$
t \leq \tau \text {, }
$$

where $t=\tau$ is a limiting condition, we use the hyperbolic functional identities to obtain,

$$
\begin{aligned}
& \cosh (\sigma)=\frac{1}{\sqrt{1-\tanh ^{2}(\sigma)}}=\frac{1}{\sqrt{1-t^{2} / \tau^{2}}}, \\
& \sinh (\sigma)=\frac{\tanh (\sigma)}{\sqrt{1-\tanh ^{2}(\sigma)}}=\frac{t / \tau}{\sqrt{1-t^{2} / \tau^{2}}} .
\end{aligned}
$$


Substituting from (47) and (48) into (42) and (43) we obtain

$$
\begin{aligned}
r^{\prime} & =\frac{r-v t}{\sqrt{1-t^{2} / \tau^{2}}}, \\
\tau v^{\prime} & =\frac{\tau v-r t / \tau}{\sqrt{1-t^{2} / \tau^{2}}} .
\end{aligned}
$$

By inverting (49) and (50) we get the inverse transform equations

$$
\begin{aligned}
r & =\frac{r^{\prime}+v^{\prime} t}{\sqrt{1-t^{2} / \tau^{2}}}, \\
\tau \nu & =\frac{\tau v^{\prime}+r^{\prime} t / \tau}{\sqrt{1-t^{2} / \tau^{2}}} .
\end{aligned}
$$

It can be verified that the transformations (49)-(52) satisfy the invariance requirement of (41) that $S^{2}=S^{\prime 2}$ by direct substitution into (36) and (37). As was previously mentioned, for a galaxy $O$ with 4-vector $S=(\tau v, r)$ observed by the observer in frame $K$, where $S^{2}=\tau^{2} v^{2}-r^{2}=0$, the transformation Equations (49) and (50) gives for the galaxy $O$ observed in $K^{\prime}$ the 4-vector $S^{\prime}=\left(\tau v^{\prime}, r^{\prime}\right)$ where, by the invariance of the transformation, $S^{\prime 2}=\tau^{2} v^{\prime 2}-r^{\prime 2}=0$. Thus, Hubble coordinates are conserved. In the next section we will use this property to obtain the cosmological redshift relation.

\section{Cosmological Redshift of Light}

We wish to quantify observations of light wave phenomena in the expanding universe made by observers at different cosmic times. Consider the distance $r$ to a galaxy $O$ which is in the Hubble flow, measured by the observer at the origin of $K$. For the observer at the origin of $K^{\prime}$ the distance to the same galaxy $O$ is $r^{\prime}$. If $r=N \lambda$ and $r^{\prime}=N \lambda^{\prime}$, where the $\lambda^{\prime}$ s are the measured wavelengths of the light from the galaxy and $N$ is the fixed number of wavelengths, then taking the ratio of distances we get

$$
\frac{r}{r^{\prime}}=\frac{N \lambda}{N \lambda^{\prime}}=\frac{\lambda}{\lambda^{\prime}}=1+z,
$$

where $z$ is the cosmological redshift of the light due to the expansion of space during the cosmic time $t$ between frames $K^{\prime}$ and $K$. Substituting for $r$ from (51) into (53) gives

$$
\begin{aligned}
\frac{r}{r^{\prime}} & =\frac{\left(r^{\prime}+t v^{\prime}\right) / r^{\prime}}{\sqrt{1-t^{2} / \tau^{2}}}, \\
& =\frac{1+t v^{\prime} / r^{\prime}}{\sqrt{1-t^{2} / \tau^{2}}} .
\end{aligned}
$$

For a galaxy which is in the Hubble flow,

$$
\frac{v^{\prime}}{r^{\prime}}=\frac{1}{\tau}
$$

Substituting from (56) into (55), and along with (53) yields

$$
1+z=\frac{\lambda}{\lambda^{\prime}}=\frac{r}{r^{\prime}}=\frac{1+t / \tau}{\sqrt{1-t^{2} / \tau^{2}}}=\sqrt{\frac{1+t / \tau}{1-t / \tau}} .
$$

Equation (57) is the cosmological redshift of the wavelength of light measured between observers in frames $K$ and $K^{\prime}$. Inverting (57) we get

$$
\frac{t}{\tau}=\frac{(1+z)^{2}-1}{(1+z)^{2}+1}
$$




\section{Dilation of Cosmic Time Due to the Expansion of Space}

This transformation is similar to the lengthening of the wavelength of light from a distant galaxy by the factor $(1+z)$. From the cosmological redshift relation (57) and the fact that the periods $\tau_{\lambda}$ and $\tau_{\lambda^{\prime}}^{\prime}$ of a wave of light are related to the wavelengths $\lambda$ and $\lambda^{\prime}$, respectively, by

$$
\begin{aligned}
\tau_{\lambda} & =\frac{\lambda}{c}, \\
\tau_{\lambda}^{\prime} & =\frac{\lambda^{\prime}}{c},
\end{aligned}
$$

we have from (53),

$$
\frac{N \lambda / c}{N \lambda^{\prime} / c}=\frac{N \tau_{\lambda}}{N \tau_{\lambda}^{\prime}}=\frac{T}{T^{\prime}}=1+z,
$$

where $T=\tau_{\lambda}$ and $T^{\prime}=\tau_{\lambda}^{\prime}$. Then the cosmic time dilation from (61) is given by

$$
T=T^{\prime}(1+z),
$$

where we assume that $T^{\prime}$ is an arbitrary time interval in $K^{\prime}$.

\section{Relativity of Cosmic Time}

Dividing (51) by (52) we obtain the transformation for the addition of cosmic time from $K^{\prime}$ to $K$, analogous to the addition of velocities in SR,

$$
t_{2}=\frac{r}{v}=\frac{r^{\prime}+t v^{\prime}}{v^{\prime}+t r^{\prime} / \tau^{2}}=\frac{t_{1}^{\prime}+t}{1+t t_{1}^{\prime} / \tau^{2}},
$$

where $t_{1}^{\prime}=r^{\prime} / v^{\prime}$. The inverse transformation, from $K$ to $K^{\prime}$ is obtained by dividing (49) by (50) giving

$$
t_{1}^{\prime}=\frac{r^{\prime}}{v^{\prime}}=\frac{r-t v}{v-t r / \tau^{2}}=\frac{t_{2}-t}{1-t t_{2} / \tau^{2}},
$$

where $t_{2}=r / v$. We refer to (63) and (64) as the general cosmic time addition relations.

Setting $t_{1}^{\prime}=\tau$ in (63) yields

$$
t_{2}=\frac{\tau+t}{1+t \tau / \tau^{2}}=\tau,
$$

implying that two cosmic times can never add up to more than $\tau$. The identical result is obtained from (64) for the time $t_{1}^{\prime}$ when $t_{2}=\tau$.

\subsection{Contraction of a Small Interval of Cosmic Time in the Past}

An increase in cosmic time $t$ by $\Delta t^{\prime}$ in frame $K^{\prime}$ at cosmic time $t$, where $\Delta t^{\prime} \ll \tau$, will have a value $t+\Delta t$ for the observer in $K$ at cosmic time 0 given by the law of addition of cosmic times (63) by setting $t_{1}^{\prime}=\Delta t^{\prime}$ and $t_{2}=t+\Delta t$,

$$
t+\Delta t=\frac{t+\Delta t^{\prime}}{1+t \Delta t^{\prime} / \tau^{2}}
$$

which yields

$$
\Delta t=\frac{t+\Delta t^{\prime}}{1+t \Delta t^{\prime} / \tau^{2}}-t
$$




$$
\begin{gathered}
=\frac{t+\Delta t^{\prime}-t\left(1+t \Delta t^{\prime} / \tau^{2}\right)}{1+t \Delta t^{\prime} / \tau^{2}}, \\
\approx \Delta t^{\prime}\left(1-t^{2} / \tau^{2}\right),
\end{gathered}
$$

since $\Delta t^{\prime} \ll \tau$. This is a cosmic time contraction of a small time $\Delta t^{\prime}$ in $K^{\prime}$ at cosmic time $t$ measured by the observer in $K$ [10]. It implies that when viewed from the present epoch, a clock will appear to tick more slowly the further back it is in cosmic time. However, this does not alter the physical constants like $G$, $\hbar$, or $e$, which remain constants. This is analogous to the case in SR where a clock at the origin of a frame with relative velocity $v$ to the local frame, ticks at the rate $\delta t^{\prime}$ in its own frame but appears to run more slowly at the rate $\delta t^{\prime} / \sqrt{1-v^{2} / c^{2}}$ when viewed from the local frame, but the physical constants do not vary.

\subsection{Dilation of a Small Interval of Cosmic Time in the Present}

There is a second kind of effect of time addition which is measured by the observer in $K^{\prime}$ situated at cosmic time $t$ from $K$. Take the cosmic time lapse $\Delta t=(t+\Delta t)-t$ recorded in $K$ where $\Delta t \ll \tau$. What is the observation of that time lapse for the observer in $K^{\prime}$ ? In other words, in $K^{\prime}$ what is the difference of the later time $t+\Delta t$ with respect to the earlier time $t$ ? The time transformation we use for the $K^{\prime}$ frame is from (64) by setting $t_{1}^{\prime}=\Delta t^{\prime}, \quad t_{2}=t+\Delta t$ giving

$$
\begin{aligned}
\Delta t^{\prime} & =\frac{(t+\Delta t)-t}{1-t(t+\Delta t) / \tau^{2}}, \\
& \approx \frac{\Delta t}{1-t^{2} / \tau^{2}},
\end{aligned}
$$

since $\Delta t \ll \tau$. This is a cosmic time dilation of a small time interval in frame $K$ measured in $K^{\prime}$. It infers that a short time interval at the present epoch corresponds to a larger time interval further back in cosmic time. Note that if $t+\Delta t=\tau$ then (70) gives $\Delta t^{\prime}=\tau$ so that we never get a time greater than $\tau$ as long as we add times $\leq \tau$.

\section{Total Cosmic Time Transformation Due to the Expansion of Space and the Additon of Cosmic Times}

Combine the two transformations by taking the product of time dilation (62) due to the expansion of the universe with time contraction (69) due to the addition of cosmic times. The total elapsed cosmic time $\Delta t$ observed by the observer in $K$ at cosmic time 0 for a small time change $\Delta t^{\prime} \ll \tau$ in frame $K^{\prime}$ at cosmic time $t$ is given by

$$
\Delta t=(1+z)\left(1-t^{2} / \tau^{2}\right) \Delta t^{\prime}=g_{1}(t) \Delta t^{\prime},
$$

where by substituting for $1+z$ from (57), the cosmic aging function $g_{1}(t)$ is defined by

$$
g_{1}(t)=\left(\sqrt{\frac{1+t / \tau}{1-t / \tau}}\right)\left(1-t^{2} / \tau^{2}\right) .
$$

Substituting for $t / \tau$ from (58) in terms of redshift $z$ into (73) yields,

$$
g_{1}(z)=\frac{4(1+z)^{3}}{\left[(1+z)^{2}+1\right]^{2}} .
$$

The cosmic aging function $g_{1}(t)>1$ for $t / \tau<0.8392$, which gives a time dilation, and $g_{1}(t)<1$ for $t / \tau>0.8395$, which corresponds to a time contraction. The maximum occurs at $t / \tau=1 / 2$ where $g_{1}(\tau / 2)=1.299$ which, from (57), corresponds to a redshift $z \approx 0.732$. Figure 1 is a plot of the cosmic aging function $g_{1}(z)$. 


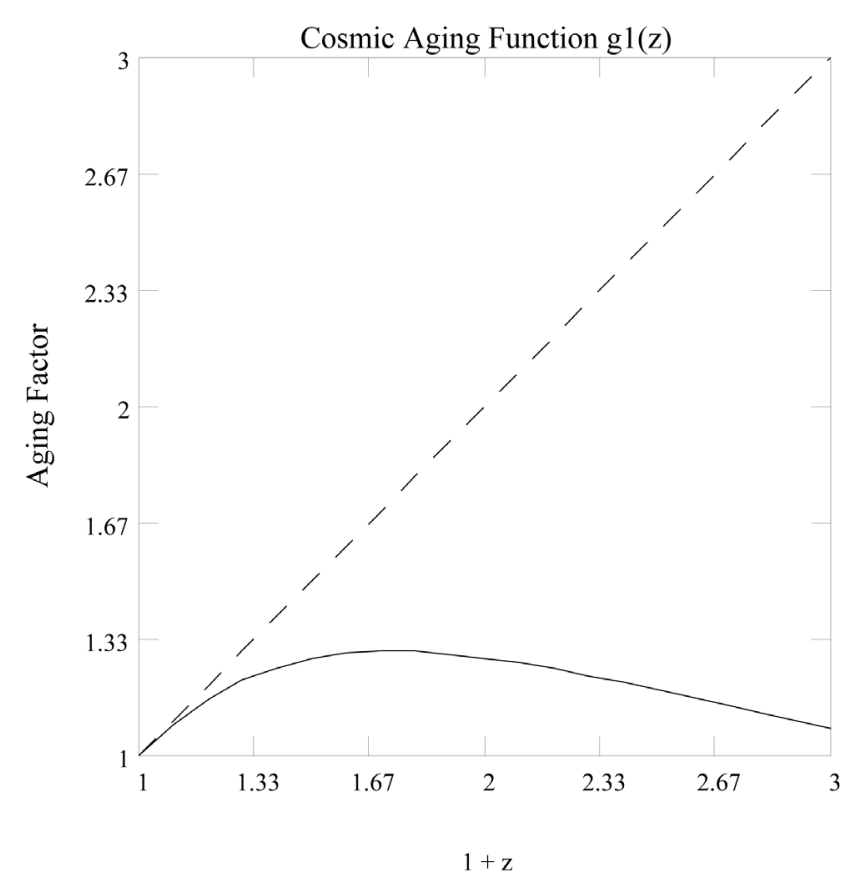

Figure 1. Cosmic aging function $g_{1}(z)=4(1+z)^{3} /\left[(1+z)^{2}+1\right]^{2}$,

$0<z<2$, solid line. The dashed line is $1+z$.

\section{Distances}

We use the distance relation (23) with the density $\Omega=\Omega_{M}$, giving

$$
r=\frac{c \tau \sinh \left((v / c) \sqrt{1-\Omega_{M}}\right)}{\sqrt{1-\Omega_{M}}}
$$

where $\Omega_{M}$ is the average mass density parameter at the present epoch.

In CGR the luminosity distance relation $D_{L}$ includes the contraction of a small interval of cosmic time in the past (69). It enters through the concept that the energy measured over an interval of time $\Delta t^{\prime}$ from a source with luminosity $L$ at rest in frame $K^{\prime}$ at cosmic time $t$, radiates a proportional quantity of energy $E$ measured by the observer in $K$ given by

$$
E \propto L\left(1-t^{2} / \tau^{2}\right)(1+z) \Delta t^{\prime}=L g_{1}(t) \Delta t^{\prime},
$$

which from (73) is the cosmic aging function $g_{1}(t)$ operating on the time interval $\Delta t^{\prime}$. Using (76) as the form for the energy of the source in the derivation [10], the luminosity distance $D_{L}$ is given by

$$
D_{L}=\frac{r(1+z)}{\sqrt{1-t^{2} / \tau^{2}}},
$$

which is a factor $\left(1-t^{2} / \tau^{2}\right)^{-1 / 2}$ of the standard form $D_{L}=r(1+z)$, implying that in CGR sources appear less luminous and thus further away due to the relativity of cosmic time. Substituting for distance $r$ from (75) and for $1+z$ from (57), and simplifying, (77) becomes

$$
D_{L}(t)=\frac{c \tau \sinh \left((v / c) \sqrt{1-\Omega_{M}}\right)}{(1-t / \tau) \sqrt{1-\Omega_{M}}} .
$$

In practice we make the substitution $t / \tau=v / c$, [4] (Appendix B. 4.2). 


\section{Distance Data Fitting}

We apply the luminosity distance relation (78) plus a calculated best fit fixed offset $a_{\text {off }}$ to get the apparent magnitude $m(z)$ of a distant luminous source,

$$
m(z)=5 \log \left[D_{L}(z)\right]+M_{B}+a_{\text {off }},
$$

where we apply (58) to convert from $t / \tau$ to $z$ in obtaining $D_{L}(z)$. We use the CGR standard value [9] of $c \tau=4158 \mathrm{Mpc}$. In practice the source absolute magnitude $M_{B}$ is absorbed into the value of the offset $a_{\text {off }}$. We include in our analysis data from both SNE-Ia and GRB studies. The SNE-Ia data come from the Supernova Cosmology Project SCP Union 2.1 data set [5] of 580 SNe-Ia magnitudes and errors up to $z<1.5$. The GRB distance data of 69 burst events come from [6] which are selected events up to $z<7$ from website data provided by [11]. For the $m_{j}$ observed magnitudes and merr $_{j}$ respective errors, the $\chi^{2}$ for the fit is defined by

$$
\chi^{2}=\sum_{j=1}^{N}\left[\frac{m_{j}-m\left(z_{j}\right)}{m e r r_{j}}\right]^{2} .
$$

The reduced chi-squared $\chi_{\text {red }}^{2}$ is given by

$$
\chi_{\text {red }}^{2}=\frac{\chi^{2}}{(N-k-1)},
$$

where $N$ is the number of data samples and $k$ is the number of fitting parameters.

For the $\Lambda C D M$ model the luminosity distance relation is given by

$$
D_{L \lambda c d m}(z)=c \tau(1+z) \int_{0}^{z} \frac{\mathrm{d} u}{\sqrt{\Omega_{M}(1+u)^{3}+\Omega_{\Lambda}}},
$$

where $\Omega_{M}+\Omega_{\Lambda}=1$ for flat space [12].

Although SNE-Ia data are independent of any particular cosmology, this is not so for GRB data which must be calibrated with a specified cosmological model. This is because SNE-Ia have nearby sources to use for calibration, but for GRB there are no nearby sources for this purpose. The original GRB data set was calibrated with the $\Lambda C D M$ cosmology. To recalibrate the GRB data for the CGR cosmological model [13] we use the relation

$$
M_{c g r}(z)=M_{\lambda c d m}(z)+\log \left[\frac{\gamma_{1} D L(z)}{D L_{\lambda c d m}(z)}\right],
$$

where $M_{\lambda c d m}$ is the original GRB distance modulus calibrated with the $\Lambda C D M$ model, $M_{c g r}(z)$ is the distance modulus calibrated for the CGR model and $\gamma_{1}$ is a conversion factor. The magnitude errors were also converted using (83). We determined a good fitting value of $\gamma_{1}=1.044442$ which was used to convert the GRB distance moduli for all fits to the CGR model. The converted GRB data were combined with the SNE-Ia data to form the complete data set. For the CGR model, the number of parameters $k=3$ for $\Omega_{M}$, $a_{\text {off }}$ and $\gamma_{1}$. The parameter $\tau$ is fixed in this analysis, so the number of degrees of freedom $N_{\text {dof }}=N-k-1=645$, the same as for the $\Lambda C D M$ model. The best fit for the CGR model is shown in Table 1 for $\Omega_{M}=0.800 \pm 0.080$ (a conservative estimate of $\pm 10 \%$ error) with offset $a_{\text {off }}=0.140$ and $\gamma_{1}=1.044442$ having a reduced chi-squared $\chi^{2} / N_{\text {dof }}=1.001182$ for $N_{\text {dof }}=645$. The results are shown in Figure 2. Figure 3 shows the histogram of the normalized residual errors for the fit. The solid curve is a Gaussian with mean $\mu=0$ and standard deviation $\sigma=1$ with an amplitude $A=135$ estimated "by eye" to give a close fit to the histogram. The fit appears good [6] [13] [14].

We fit the $\Lambda C D M$ cosmology to the original GRB data set, which was calibrated with $\Omega_{M}=0.270$ and $\Omega_{\Lambda}=0.730$. We assume $k=3$ parameters in the model, for $\Omega_{M}, \Omega_{\Lambda}$ and $a_{\text {off }}$. We use a fixed Hubble 


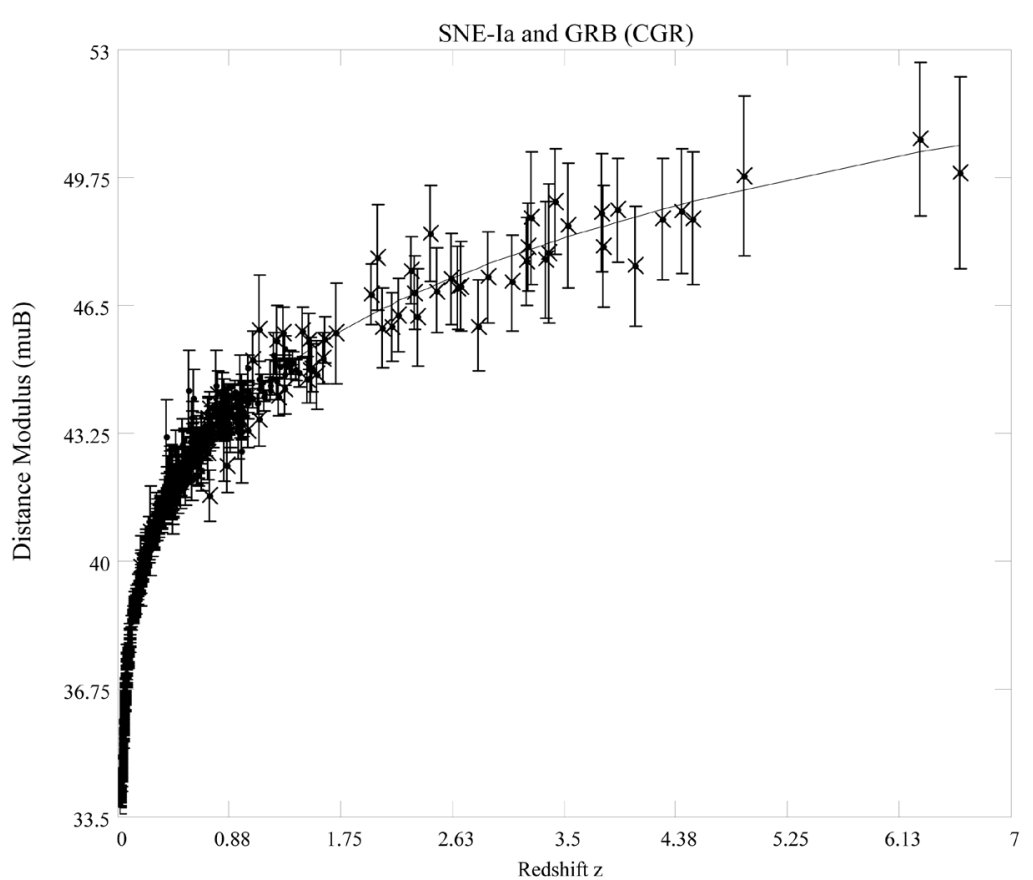

Figure 2. SCP Union 2.1 SNe-Ia data [5] (filled circles) and GRB [6] (dotted x's). The solid line is for CGR $D L(z)$ from (79). The CGR standard value was used for $1 / \tau=h=72.2 \pm 0.84 \mathrm{~km} / \mathrm{s} / \mathrm{Mpc}$. The parameters for the CGR model were the calculated best fit values, with mass density $\Omega_{M}=0.800$, offset $a_{\text {off }}=0.140$ and GRB conversion factor $\gamma_{1}=1.044442$. Magnitude and magnitude errors both were converted to the CGR model (83). For the fit to 649 data points with 3 parameters the reduced $\chi^{2}=1.001182$.

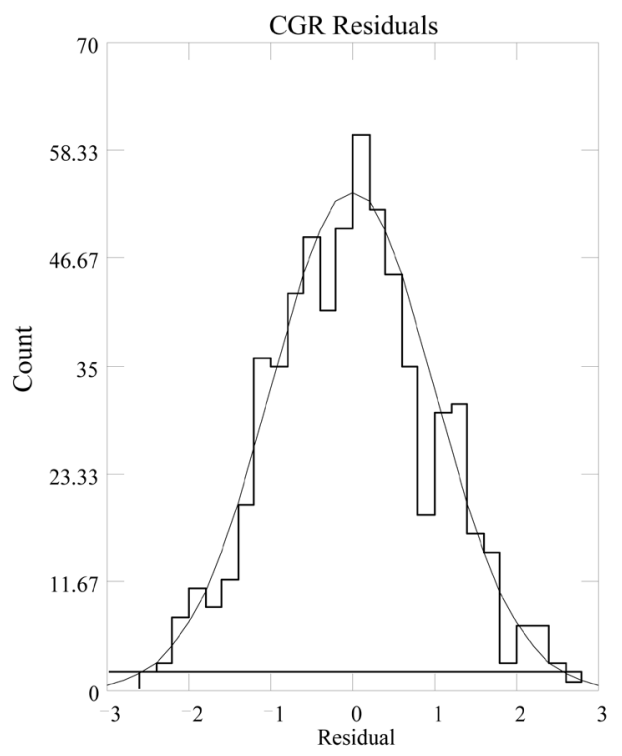

Figure 3. Histogram of CGR residuals $m_{k}-m\left(z_{k}\right)$ with $D L\left(z_{k}\right)$ from (79) with redshift $z_{k}$ from the SCP Union 2.1 SNe-Ia data [5] and GRB data [6], for calculated best fits with mass density $\Omega_{M}=0.800$, offset $a_{\text {off }}=0.140$ and $\gamma_{1}=1.044442$. The solid line is a standard Gaussian with mean $\mu=0$ and standard deviation $\sigma=1$ and the amplitude is scaled by the factor $A=135$ to give a good "by eye" fit to the histogram. 
Table 1. CGR vs. $\Lambda C D M$ model performances with reduced $\chi^{2} /(n-k-1)$. The number of samples $n=649$, and number of parameters $k=3$ for both models which gives $n-k-1=645$. The CGR row showing the best fit is marked with a ( ${ }^{*}$ ). Refer to the text for an explanation of the best fitting model. The $\Lambda C D M$ model, with fixed $\Omega_{M}$ and fixed $\Omega_{\Lambda}$ has a single best fit for $a_{\text {off }}$ based on the original data.

\begin{tabular}{ccccc}
\hline Model & $\Omega_{M}$ & $a_{\text {off }}$ & $\chi^{2}$ & $\chi^{2} / 645$ \\
\hline CGR & 0.650 & 0.140 & 640.656 & 0.993265 \\
CGR & 0.700 & 0.140 & 642.143 & 0.995571 \\
CGR & 0.750 & 0.140 & 643.844 & 0.998208 \\
* CGR & 0.800 & 0.140 & 645.762 & 1.001182 \\
CGR & 0.850 & 0.143 & 648.263 & 1.005059 \\
CGR & 0.900 & 0.145 & 650.815 & 1.009015 \\
$\Lambda$ CDM & 0.270 & 0.060 & 635.397 & 0.985111 \\
\hline
\end{tabular}

constant of $H_{0}=1 / \tau=72.2 \mathrm{~km} \cdot \mathrm{s}^{-1} \cdot \mathrm{Mpc}^{-1}$. The best fit occurred for offset $a_{\text {off }}=0.060$. Figure 4 shows the Hubble Diagram for the fit of $D_{L \lambda c d m}(z)$ to the combined data, with the GRB moduli used unaltered from the data set. The parameter $H_{0}$ is fixed in this analysis, so the number of degrees of freedom is the same as for the CGR model. The reduced chi-squared $\chi^{2} / N_{\text {dof }}=0.985111$. Figure 5 shows the histogram of the normalized residual errors for the fit. The solid curve is a Gaussian with mean $\mu=0$ and standard deviation $\sigma=1$ with an amplitude $A=135$ taken from the CGR histogram.

Under the reduced chi-squared statistical model, the ideal reduced $\chi^{2}=1$ with the errors distributed normally (Gaussian) about $\mu=0$ with $\sigma=1$. The model with a reduced $\chi^{2}$ which is closest to 1 is preferred. Models with reduced $\chi^{2}>1$ are deemed to have too few parameters, so "under fit" the data. Models with reduced $\chi^{2}<1$ are deemed to have too many parameters and thus "over fit" the data.

We see that the CGR model under fits the data by $\approx 0.001182$, while the $\Lambda C D M$ model over fits the data by $\approx 0.014889$. For this analysis, the CGR model is $\approx 10$ times better at fitting the combined data. However, considering that we did not vary the mass densities $\Omega_{M}$ and $\Omega_{\Lambda}$, nor the Hubble parameter $H_{0}$, when fitting with the $\Lambda C D M$ model, we make this conclusion as mainly a statement of our confidence in the CGR model. A more rigorous analysis of the fitting operation would be required.

\section{Dark Matter and the X Particle Hypothesis}

Assume that the mass density $\Omega_{M}=\Omega_{B}+\Omega_{D}$, that is, composed of baryonic matter $\Omega_{B}$ and cold dark matter $\Omega_{D}$. For $\Omega_{B} h^{2} \approx 0.020 \pm 0.002$ (95\% confidence level) [15], with the CGR value $h=0.722$, this gives

$$
\Omega_{B} \approx 0.038 \pm 0.004 \text {. }
$$

Then, for $\Omega_{M c g r}=0.800 \pm 0.080$ and $\Omega_{M \lambda c d m}=0.270 \pm 0.013$, the CGR model gives a dark matter density of

$$
\Omega_{\text {Dcgr }} \approx 0.762 \pm 0.084
$$

compared to the $\Lambda C D M$ model which gives a dark matter density of

$$
\Omega_{\text {Dicdm }} \approx 0.232 \pm 0.017 \text {. }
$$

In an extension to the standard model (SM), a hypothetical $X$ particle [8] is theorized to exist, having 2 species, $X_{1}$ and $X_{2}$ (and their conjugate species $\bar{X}_{1}$ and $\bar{X}_{2}$.) These particles were generated non-thermally during the early universe. The $X_{1}$ decays either into a visible three quark state $(U D D)$, or the hidden state $(Y, \Phi)$, with each state having baryon number +1 . The conjugate state $\bar{X}_{1}$ decays to the visible three quark state ( $\bar{U} \bar{D} \bar{D})$ or the hidden state $\left(\bar{Y}, \Phi^{*}\right)$, with each state having baryon number -1 . All of the dark matter today, in 


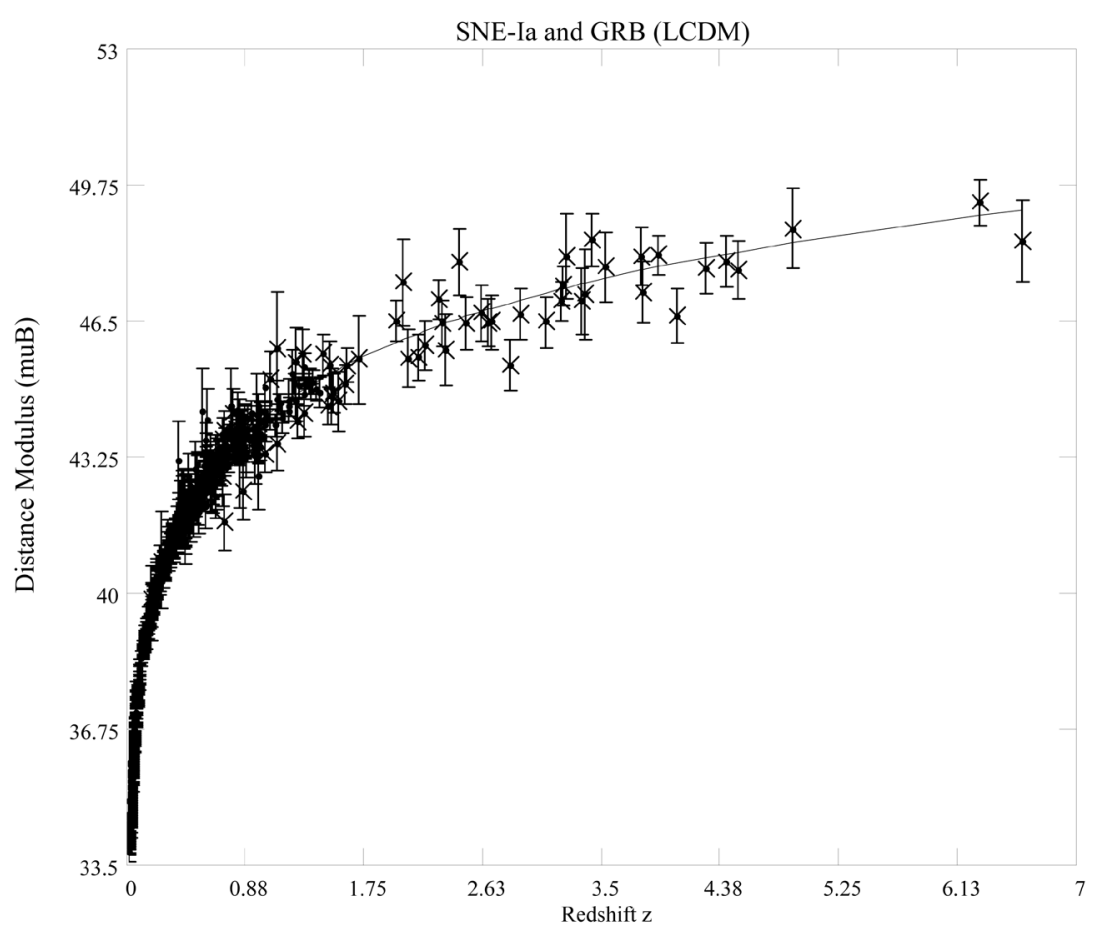

Figure 4. SCP Union 2.1 SNe-Ia data [5] (filled circles) and GRB [6] (dotted x's). The solid line is for $D L_{\lambda c d m}(z)$ from (82). The CGR standard value was used for $1 / \tau=h=72.2 \pm 0.84 \mathrm{~km} / \mathrm{s} / \mathrm{Mpc}$. For the $\Lambda C D M$ model, with fixed $\Omega_{M}=0.270$ and fixed $\Omega_{\Lambda}=0.730$, the calculated best fit value for offset $a_{\text {off }}=0.060$. For the fit to 649 data points with 3 parameters the reduced $\chi^{2}=0.985111$.

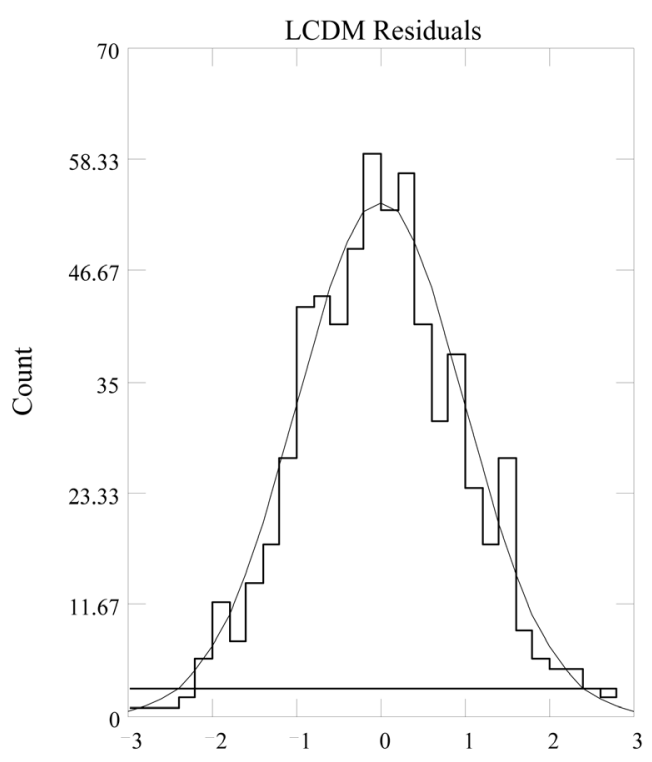

Figure 5. Histogram of $\Lambda C D M$ residuals $m_{k}-m\left(z_{k}\right)$ for $D L_{\lambda c d m}\left(z_{k}\right)$ from (82) with redshift $z_{k}$ from the SCP Union 2.1 SNe-Ia data [5] and GRB data [6], for fixed mass density $\Omega_{M}=0.270$ and fixed dark energy mass density $\Omega_{\Lambda}=0.730$, with a calculated best fit offset $a_{\text {off }}=0.060$. The solid line is a standard Gaussian with mean $\mu=0$ and standard deviation $\sigma=1$ and the amplitude factor $A=135$ comes from the CGR histogram. 
this extended model, is theorized to be composed entirely of hidden particle states $\left(\bar{Y}, \Phi^{*}\right)$. Rare processes can transfer baryonic number from the hidden sector to the visible sector through inelastic scattering of anti-baryonic dark matter states $\left(\bar{Y}, \Phi^{*}\right)$, annihilating baryons in the visible sector. The cosmic abundance of remnant $\bar{Y}$ and $\Phi^{*}$ particles, with densities given by $n_{\bar{Y}}$ and $n_{\Phi^{*}}$, respectively, is the same as the baryon density $n_{B}$ in the universe today, and thus would have the same abundance ratio as $\eta_{B}$, the baryon to photon $\left(n_{\gamma}\right)$ ratio. That is,

$$
\frac{n_{\bar{Y}}}{n_{\gamma}}=\frac{n_{\Phi^{*}}}{n_{\gamma}}=\frac{n_{B}}{n_{\gamma}}=\eta_{B} \approx 6 \times 10^{-10}
$$

for a baryon density $\Omega_{B} \approx 0.04$. Further details of this extension to SM is beyond the scope of this paper. From [8] (Equation (10)) we can relate the ratio of the density of dark matter (anti-baryonic) to baryonic matter in the universe to the ratio of the rest masses of the $\bar{Y}, \Phi^{*}$ and proton by

$$
\frac{\Omega_{D}}{\Omega_{B}}=\frac{m_{\bar{Y}}+m_{\Phi^{*}}}{m_{p}} \approx \frac{2 w m_{p}}{m_{p}}=2 w,
$$

where $m_{\bar{Y}}$ and $m_{\Phi^{*}}$ are, respectively, the $\bar{Y}$ and $\Phi^{*}$ particle rest masses and $m_{p}$ is the proton rest mass and we assumed that $m_{\bar{Y}} \approx m_{\Phi^{*}} \approx w m_{p}$. For the CGR model, (88) yields

$$
\frac{\Omega_{D c g r}}{\Omega_{B}}=\frac{0.762 \pm 0.084}{0.038 \pm 0.004}=2 w_{c g r},
$$

which implies

$$
w_{c g r}=10.43 \pm 2.18 \text {. }
$$

This gives rest mass energies for the $\bar{Y}$ and $\Phi^{*}$ particles of

$$
m_{\overline{\mathrm{Y}} c g r} c^{2} \approx m_{\Phi^{*} c g r} c^{2} \approx(9.79 \pm 0.47) \mathrm{GeV} .
$$

For the $\Lambda C D M$ model, (88) yields

$$
\frac{\Omega_{D \lambda c d m}}{\Omega_{B}}=\frac{0.232 \pm 0.017}{0.038 \pm 0.004}=2 w_{\lambda c d m},
$$

which implies

$$
w_{\lambda c d m}=2.61 \pm 0.55 \text {. }
$$

This gives rest mass energies for the $\bar{Y}$ and $\Phi^{*}$ particles [8] of

$$
m_{\bar{Y} \lambda c d m} c^{2} \approx m_{\Phi^{*} \lambda c d m} c^{2} \approx(2.45 \pm 0.47) \mathrm{GeV} .
$$

In both cases above we have tried to account for the constraint that $\left|m_{\bar{Y}}-m_{\Phi^{*}}\right|<m_{p}+m_{e}$, where $m_{e}$ is the electron mass, by restricting the range of values to be within $\pm 0.47 \mathrm{GeV}$. This may be only an approximate treatment, at best.

\section{Time Dilation in SNe-Ia Light Curves}

We now consider two SNe-Ia (SNe) light curve experiments [2] [3]. Common to both experiments is the stretch of the SNe light curve interval for each distant source when compared to the standard nearby (local) source. Because these studies rely on a model for what the light curve looks like in the rest frame of the source SNe, we will require the use of both kinds of cosmic time additions described above. The light curve time interval $\Delta t_{\text {obspec }}^{\prime}$ from the distant SNe will be observed to have a time transformation which is a combination of cosmic time addition in the past combined with the time effect of the expansion of space, having the observed value $\Delta t_{\text {obs }}$ which is given by (72),

$$
\Delta t_{o b s}=\Delta t_{o b s p e c}^{\prime}(1+z)\left(1-t^{2} / \tau^{2}\right) .
$$


In CSR this is the time interval that is observed from a source light curve or any time varying phenomenon at a cosmic time $t$ in the past.

On the other hand, we can describe the light curve recorded by the observer in the frame $K^{\prime}$ at cosmic time $t$ relative to our local observer in $K$ at cosmic time 0. A light curve time duration of $\Delta t_{\text {spec }}$ in $K$, from (71) for a cosmic time addition in the present, corresponds to the value $\Delta t_{\text {spec }}^{\prime}$ in $K^{\prime}$ given by

$$
\Delta t_{\text {spec }}^{\prime}=\frac{\Delta t_{\text {spec }}}{\left(1-t^{2} / \tau^{2}\right)} .
$$

If we make the assumption that

$$
\Delta t_{\text {obspec }}^{\prime}=\Delta t_{\text {spec }}^{\prime},
$$

then combining (95) with (97) yields

$$
\Delta t_{\text {obs }}=\Delta t_{\text {spec }}(1+z) .
$$

It is evident that CSR, assuming (97), is consistent with the time dilation reports showing effects of cosmic aging equivalent with $1+z$ for redshift $z$. However, to offer a different perspective on cosmic time transformation, we will show plots of the ratio $\Delta t_{\text {obs }} / \Delta t_{\text {obspec }}^{\prime}$ given by (95), instead of $\Delta t_{\text {obs }} / \Delta t_{\text {spec }}$ which was used in those reports.

For the SNe data from [2] the light curve agings are given as the light curve width $w$ and the error in the width $\sigma_{w}$, obtained directly from [2] (Table 1) for the SCP high z SNe and from [2] (Table 3) for the Calán/Tololo low $z$ SNe. Since the goal of the experiment was to normalize each light curve to a single standard light curve, we will assume that the equivalent local rest frame time is $\Delta t_{\text {spec }}=1$ which implies from (96) that $\Delta t_{\text {spec }}^{\prime}=1 /\left(1-t^{2} / \tau^{2}\right)$. The reduced observed quantity is $w$ and we will assume using (98),

$$
\Delta t_{\text {obs }}=\Delta t_{\text {spec }}(1+z)=(1+z)=w \text {. }
$$

We will use (99) to acquire the redshift $z$ and hence $t / \tau$ from the light curve rather than using the redshift from the host galaxy. The quantity we use is the ratio $\Delta t_{\text {obs }} / \Delta t_{\text {obsppec }}^{\prime}$ from (95),

$$
\frac{\Delta t_{\text {obs }}}{\Delta t_{\text {obspec }}^{\prime}}=(1+z)\left(1-t^{2} / \tau^{2}\right) \text {. }
$$

The plotted data are shown in Figure 6. For data points with $w<1$ the redshift was set to $z=0$. The plot shows the reduction of apparent light curve aging at higher redshift. This is the effect which would be seen in the observed light curve without scaling by the rest frame aging rate. The reduced chi-squared is $\chi^{2} / 57=10.8$ for the data fitted to the cosmic aging rate $g_{1}(z)$, (74).

The next SNe data are from [3]. We take the aging rates $\left(\Delta t_{\text {spec }} / \Delta t_{\text {obs }}\right)$ from [3, Table 3], in the last column, unparenthesized. We compute

$$
\frac{\Delta t_{\text {obs }}}{\Delta t_{\text {obsspec }}^{\prime}}=\left(\Delta t_{\text {obs }} / \Delta t_{\text {spec }}\right)\left(1-t^{2} / \tau^{2}\right) .
$$

The errors come from [3] (Table 3), in the last column, parenthesized. The redshifts are computed from the aging rate data instead of from the given host galaxy values. The plotted data are shown in Figure 7. Again we note the reduction in the aging rate at higher redshifts. The reduced chi-squared for the data fitted to the cosmic aging rate $g_{1}(z)$ is $\chi^{2} / 34=0.690$. In Figure 8 we show the combination of all the SNe aging data from the two reports. With 93 total data points the reduced chi-squared is $\chi^{2} / 92=6.98$.

\section{Simulation of Quasar Like Light Curve Power Spectra}

The purpose of this section is to simulate quasar like light curve power spectra to compare with the report of observed low and high redshift quasar light curve power spectra [1]. The observed light curve power 


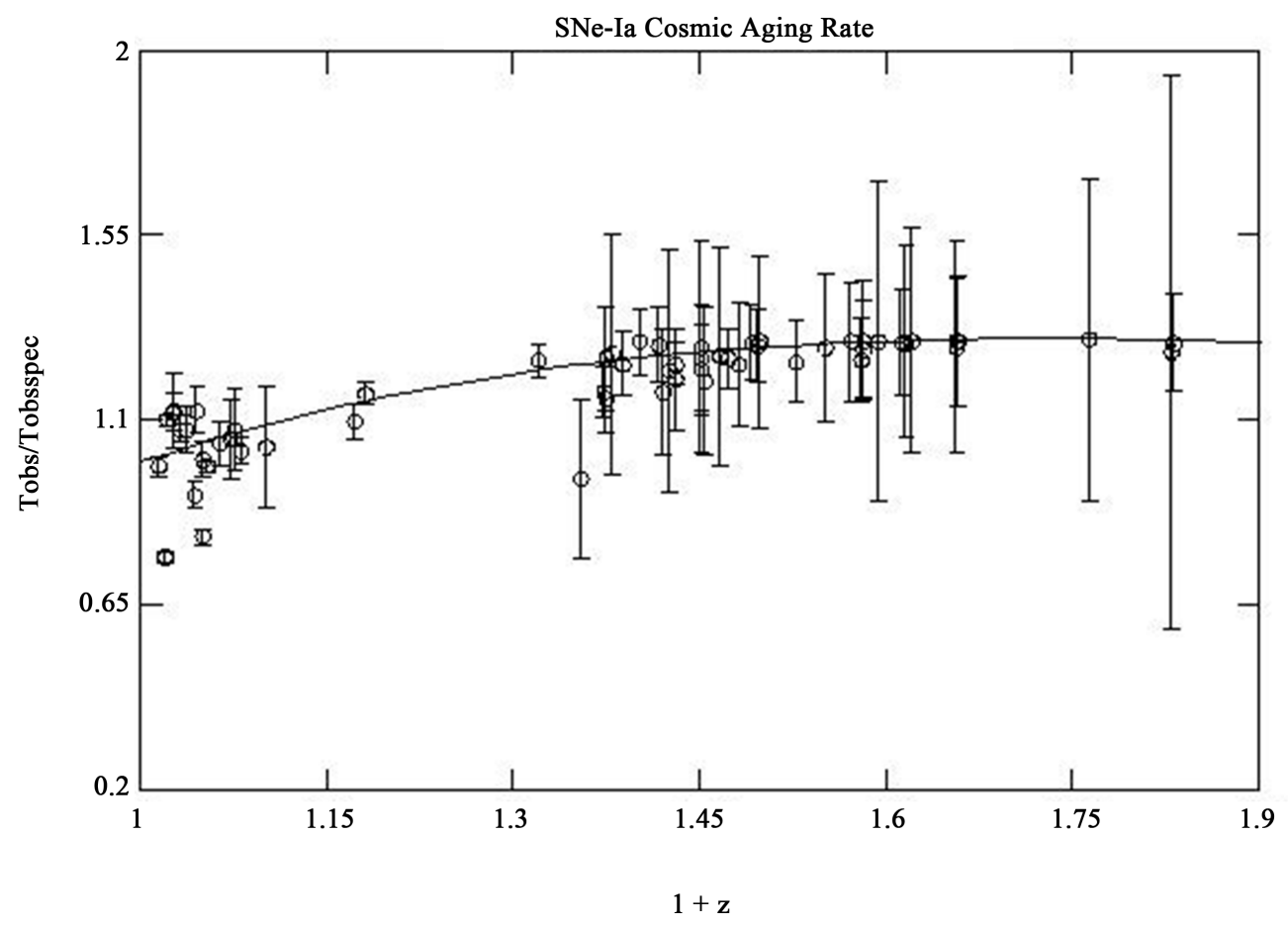

Figure 6. Calán/Tololo low $z$ and SCP high $z$ SNe-Ia light curve time intervals [2] (Goldhaber, et al.). Open circles are $\Delta t_{\text {obs }} / \Delta t_{\text {obsppec }}^{\prime}$ for each of the 58 SNe-Ia. Error bars are computed from the $\sigma_{w}$ data errors. The solid line is the cosmic aging function $g_{1}(z)$ of (74). The reduced $\chi^{2}$ for the fit of $g_{1}(z)$ to the data is $\chi^{2} / 57=10.8$.

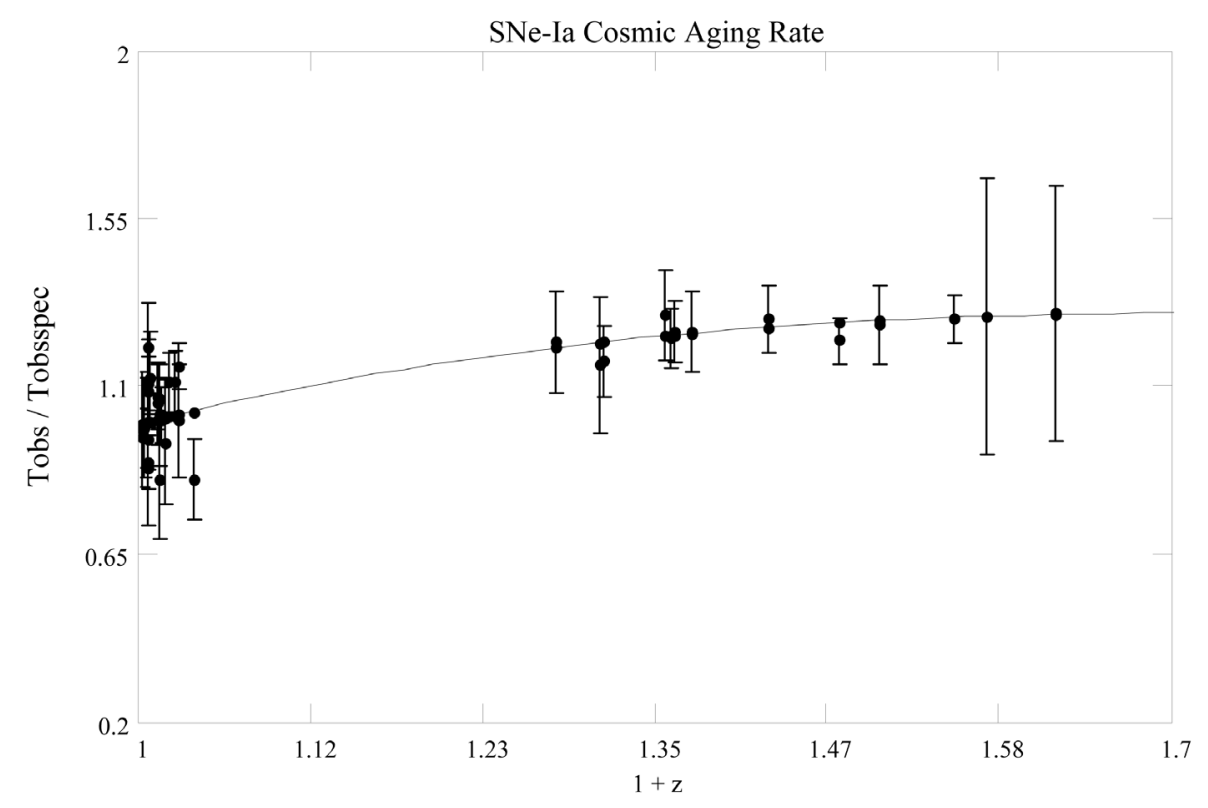

Figure 7. SCP low $z$ and high $z$ SNe-Ia light curve time intervals [3] (Blondin, et al.). Filled circles are $\Delta t_{\text {obs }} / \Delta t_{\text {obsspec }}^{\prime}$ for each of the $35 \mathrm{SNe}$-Ia. Error bars are computed from the data errors. The solid line is the cosmic aging function $g_{1}(z)$. The reduced $\chi^{2}$ for the fit of $g_{1}(z)$ to the data is $\chi^{2} / 34=0.690$. 


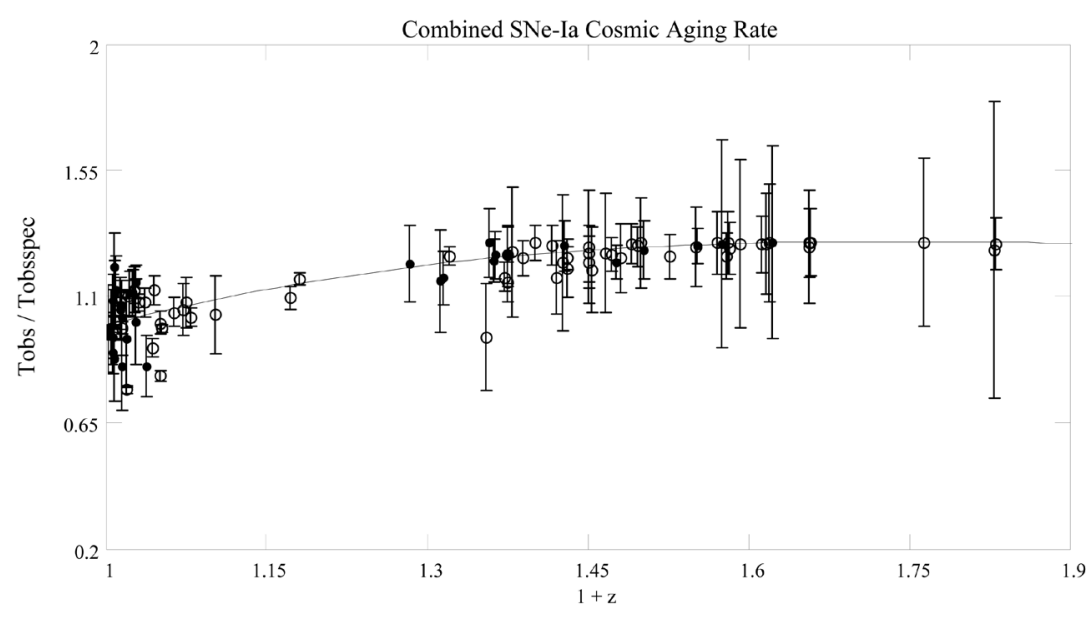

Figure 8. Combined SNe-Ia light curve time intervals $\Delta t_{\text {obs }} / \Delta t_{\text {obsspec }}^{\prime}$. Open circles are from [2] (Goldhaber, et al.). Filled circles are from [3, Blondin, et al.]. The solid line is the cosmic aging function $g_{1}(z)$. The reduced $\chi^{2}$ for the fit of $g_{1}(z)$ to the combined data is $\chi^{2} / 92=6.98$.

spectra [1] (Figure 5, left-hand panel) were found to be identical within the experimental errors. Therefore, we will assume the low and high redshift light curves are identical in the observer frame $K$. In addition, it is assumed that the redshifts are of pure cosmological origin, with no components of gravitational redshifts or Doppler shifts. In our simulation, the pseudo quasar light curve apparent magnitudes $m(j)$ at epoch $j$ is generated by the function

$$
m(j)=\exp \left(-\frac{2 \pi j}{N_{y}}\right) \cos ^{2}\left(\frac{800 f_{0} j}{N_{e}}\right) \sin ^{2}\left(\frac{200 f_{0} j}{N_{e}}\right),
$$

for each epoch $j=1,2, \cdots, N_{e}$, where $N_{e}=560, N_{y}=56$ years and $f_{0}=1 / 16.3 \mathrm{yr}=0.0613 \mathrm{yr}^{-1}$. The redshifts used are low $z=0.765$ and high $z=1.711$ and $f_{0}^{-1}=16.3 \mathrm{yr}$ are from the quasar time dilation report [1]. For better resolution we used $N_{y}=56 \mathrm{yr}$ instead of the $28 \mathrm{yr}$ which was used in the report. The Fourier power spectrum $P_{S}(z, j)$ is determined from the magnitudes $m(j)$ by [1] (Equation (1))

$$
P_{S}(z, j)=\frac{T(z)}{N_{e}}\left[\sum_{k=1, N_{e}} m(j) \cos \left(\frac{2 \pi j k}{N_{e}}\right)\right]^{2}+\frac{T(z)}{N_{e}}\left[\sum_{k=1, N_{e}} m(j) \sin \left(\frac{2 \pi j k}{N_{e}}\right)\right]^{2},
$$

where $\mathrm{j}$ runs over $N_{e}$ equally spaced epochs of simulated data separated by time $T(z) / N_{e}$. Then the time transformations will take us from the origin of observer frame $K$ to the quasar rest frame $K^{\prime}$ at cosmic time $t$. For CSR the sampling interval $T(z)$ we use is defined by

$$
T(z)=\frac{T_{0}}{g_{1}(z)},
$$

where $g_{1}(z)$ is given by (74) and

$$
T_{0}=1 / f_{0} .
$$

We divide by $g_{1}(z)$ in (104) because we are going back in time to the quasar rest frame.

For our purposes, the flat space Friedmann-Lemaitre-Robertson-Walker (FLRW) model is the CSR model with the cosmic aging function $g_{1}(z)$ replaced by $(1+z)$. For the flat space FLRW model we use for $T(z)$, 


$$
T(z)=\frac{T_{0}}{1+z},
$$

where we have again divided out the time transformation to get to the quasar rest frame.

For either model we use the fitting function $P(f, z)$ defined by [1], Equation (2)

$$
P(f, z)=\frac{C_{0}}{\left(f / f_{c}(z)\right)^{a}+\left(f / f_{c}(z)\right)^{-b}},
$$

where $C_{0}$ is the power, $f$ is the frequency, $f_{c}(z)$ is the redshift dependent frequency at maximum power and $a$ and $b$ are constants. We use the appropriate form of $f_{c}(z)$ for the CSR or the FLRW models. We show light curve power spectrum plots of $\log \left(P_{S}(z, j) \times f_{j}(z)\right)$ vs. $\log \left(f_{j}(z)\right)$ for the light curves at low and high redshift.

Assuming both quasars have identical power spectra in the observer frame $K$, we obtain the power spectrum from (103) by setting the redshift $z=0$ which is given by $P_{S}(0, j)$. This is shown in Figure 9 with the fitting power function $P_{f}(f, z)$ parameters $C_{0}=0.020524, f_{c}(z=0)=2.4 f_{0}, a=1.4 \alpha$ where $\alpha=0.81$ from [1] (Table 1, Observer frame Sample $=\mathrm{z}<1$, Index $=-0.81$ ) and $b=a$. This can be compared with [1] (Figure 5, left-hand panel).

Next we show the light curve power spectra for the low and high redshift quasars, $z_{\text {low }}=0.765$ and $Z_{\text {high }}=1.711$, respectively, as it would be observed in their rest frame. The light curves are corrected by $1 / g_{1}(z)$ since we are obtaining the light curve back in time. We show the quasar power spectrum along with the fitting function $P_{f}(f, z)$, which has the same parameters as were used at the origin of $K$ except for the frequency at maximum power which is given by

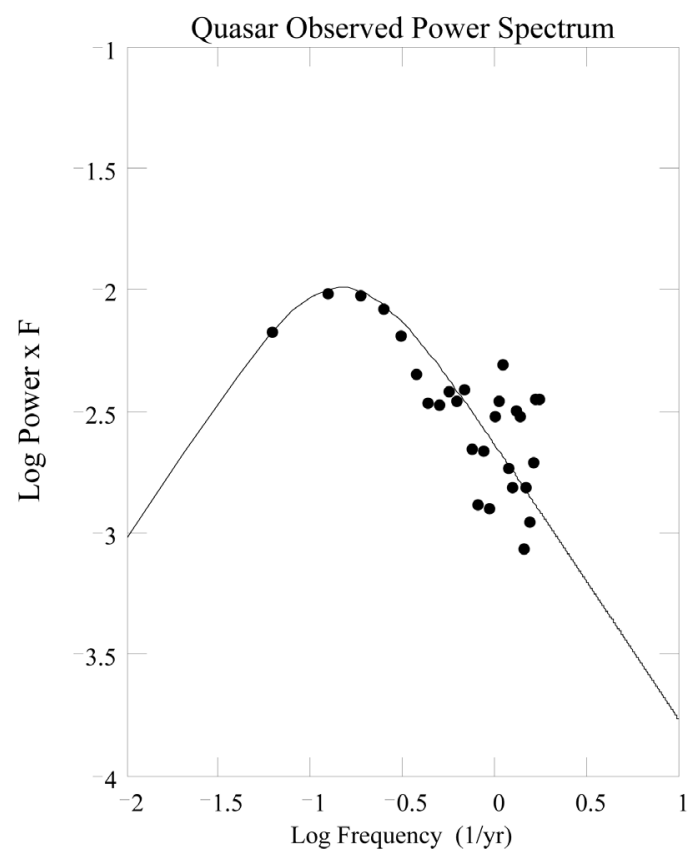

Figure 9. Simulated quasar light curve power spectrum, filled circles, observed at origin of frame $K$ at cosmic time $t=0$. Low redshift and high redshift quasar power spectra overlap so are shown as one spectrum. The frequency $f$ is $\mathrm{yr}^{-1}$. The abscissa (horizontal) axis is $\log (f)$ and the ordinate (vertical) axis is $\log ($ power $\times f)$. Both axes have unit scaling. The function $P_{f}(f)$, solid line, was fitted iteratively with parameters $C_{0}=0.020524$, $f_{c}(0)=0.14724 \mathrm{yr}^{-1}, a=1.134$ and $b=-a$, yielding $\chi^{2} / N_{y} / 2=0.042673$. 


$$
f_{c}(z)=2.4 f_{0} g_{1}(z),
$$

with $g_{1}(z)$ from (74). This is plotted in Figure 10. The fitting function has the same parameters for both the low and high redshift spectra as were used in the spectrum at the origin of $K$ except for $f_{c}(z)$.

To show what the power spectra might look like for a flat space FLRW observation we show the low and high redshift quasar light curve power spectra when the light curves are corrected for time dilation by $1 /(1+z)$, assuming a flat space cosmology. This is shown in Figure 11. This plot is similar to [1] (Figure 5, right-hand panel). The fitting function $P_{f}(f, z)$ has the same parameters for both the low and high redshift spectra as were used at the origin of $K$ except for the frequency at maximum power which is given by

$$
f_{c}(z)=2.4 f_{0}(1+z) \text {. }
$$

In Figure 12 we show a contour plot of the cosmic aging ratio $\Gamma\left(z, z^{\prime}\right)$ defined by

$$
\Gamma\left(z, z^{\prime}\right)=\frac{g_{1}(z)}{g_{1}\left(z^{\prime}\right)},
$$

between two source fields, one at redshift $z$ and the other at redshift $z^{\prime}$. This is to demonstrate that it is possible to obtain similar aging rates (eg. within $10 \%$ ) between two sources separated by large redshift. For the above low and high redshifts, $\Gamma(1.711,0.765)=0.8803$.

For another demonstration of the cosmic aging ratio, we show in Figure 13 the power spectrum for each quasar in its rest frame where $z_{1}=0.7$ and $z_{2}=1.4$. The aging ratio is $\Gamma\left(z_{2}, z_{1}\right)=0.9317$.

\section{CMB Anisotropy Acoustic Peak}

In CGR the big bang occurred at velocity $v=c$. At the recombination of protons and electrons in the baryon-photon plasma, when the photons decoupled to form the CMB radiation field, the velocity was $v=v_{r c}$,

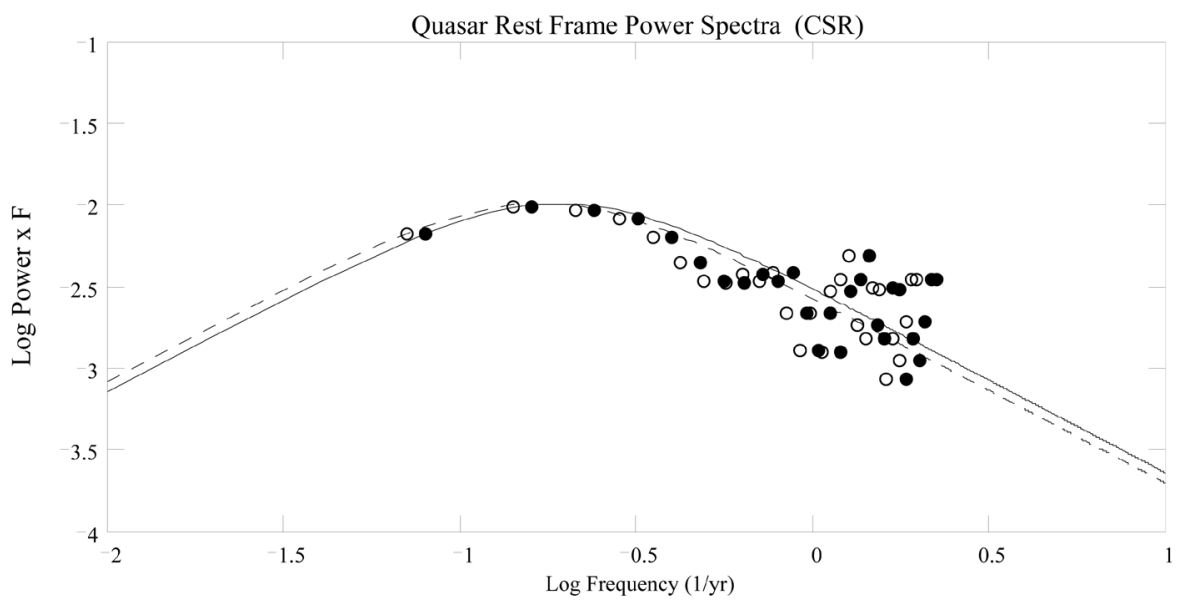

Figure 10. Simulated quasar light curve power spectra in the rest frame $K^{\prime}$ of each quasar where each light curve transforms according to CSR. The frequency $f$ is $\mathrm{yr}^{-1}$. For the low redshift power spectrum, filled circles, $z_{\text {low }}=0.765$, the horizontal axis is scaled by $g_{1}\left(z_{\text {low }}\right)$ with $g_{1}(z)$ from (74) and the vertical axis has unit scaling. The power spectrum time interval is transformed by $1 / g_{1}\left(z_{\text {low }}\right)$ and the frequency is scaled by $g_{1}\left(z_{\text {low }}\right)$ which effectively sets the scale to unity. For the high redshift power spectrum, open circles, $z_{\text {high }}=1.711$, the horizontal axis is scaled by $g_{1}\left(z_{\text {high }}\right)$ and the vertical axis has unit scaling although the power spectrum time interval is transformed by $1 / g_{1}\left(z_{\text {high }}\right)$ and the frequency is scaled by $g_{1}\left(z_{\text {high }}\right)$. The fitting function $P_{f}(f)$ has the same parameters as used at the origin of $K$ except the central frequency is scaled by $f_{c}(z)=0.14724 \times g_{1}(z)$ with $z=z_{\text {low }}$, solid line, and $z=z_{\text {high }}$, dashed line. 


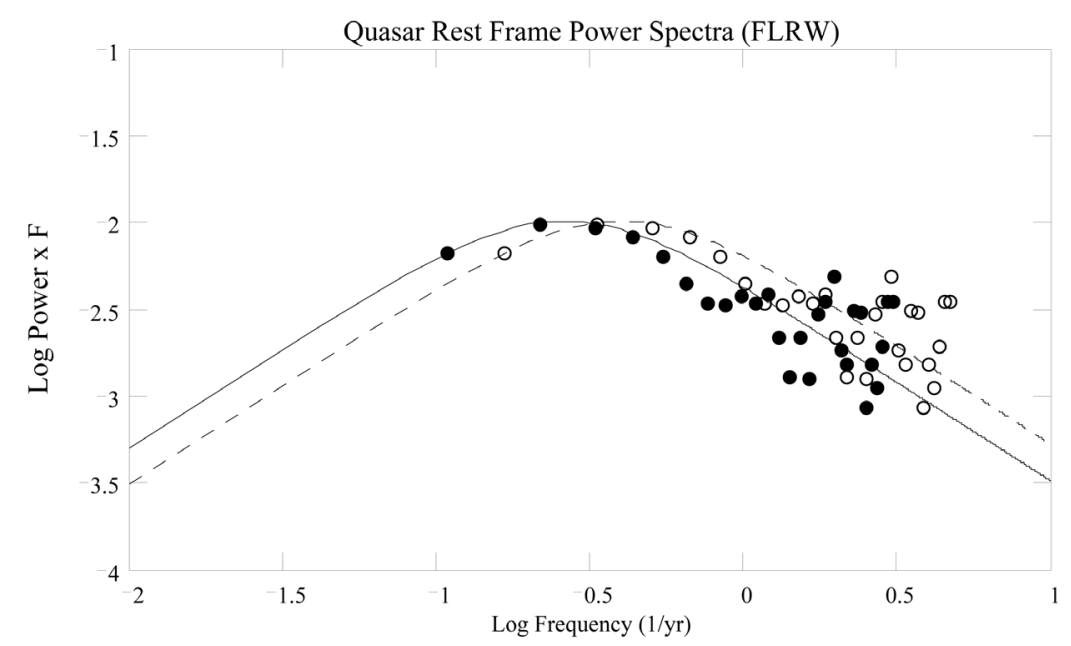

Figure 11. Simulated quasar light curve power spectra in the rest frame $K^{\prime}$ of each quasar where each light curve transforms according to flat space FLRW. The frequency $f$ is $\mathrm{yr}^{-1}$. For the low redshift power spectrum, filled circles, $z_{\text {low }}=0.765$, the horizontal axis is scaled by $1+z_{\text {low }}$. and the vertical axis has unit scaling. The power spectrum time interval is transformed by $1 / 1+z_{\text {low }}$ and the frequency is scaled by $1+z_{\text {low }}$ which effectively sets the scale to unity. For the high redshift power spectrum, open circles, $z_{\text {high }}=1.711$, the horizontal axis is scaled by $1+z_{\text {high }}$ and the vertical axis has unit scaling although the power spectrum time interval is transformed by $1 / 1+z_{\text {high }}$ and the frequency is scaled by $1+z_{\text {high }}$. The fitting function $P_{f}(f)$ has the same parameters as used at the origin of $K$ except the central frequency is scaled by $f_{c}(z)=0.14724 \times(1+z)$ with $z=z_{\text {low }}$, solid line, and $z=z_{\text {high }}$, dashed line.

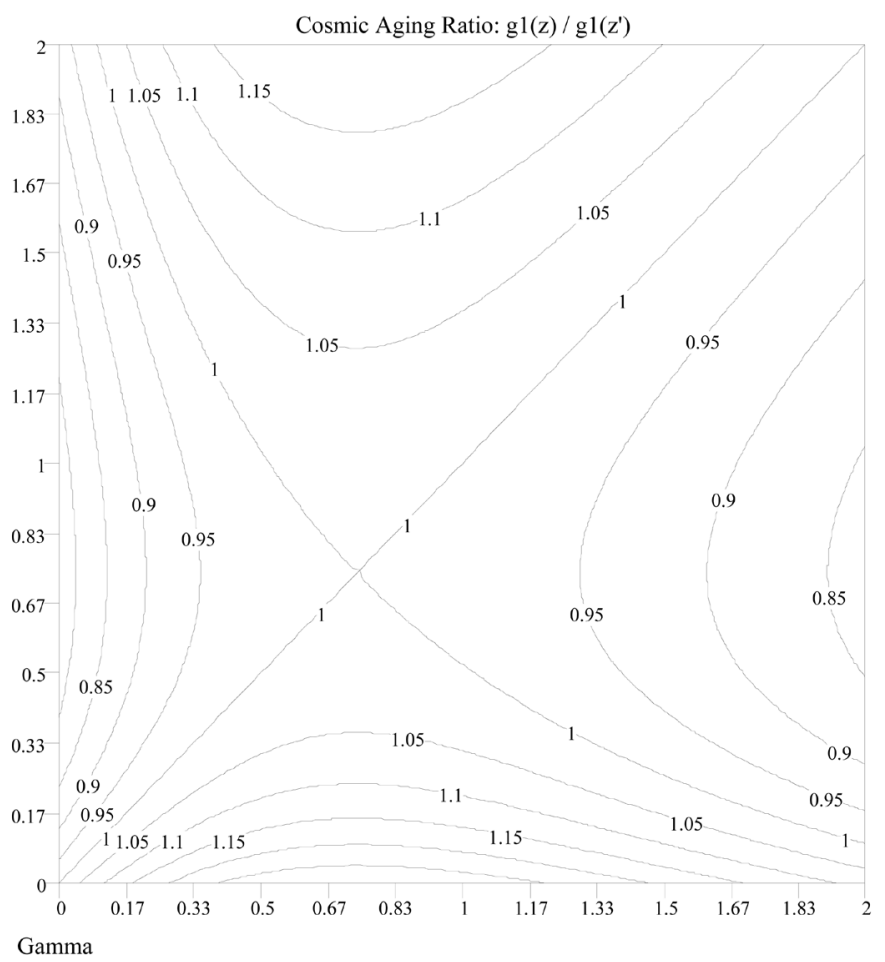

Figure 12. Contour plot of $\operatorname{Gamma}\left(z, z^{\prime}\right)=g_{1}(z) / g_{1}\left(z^{\prime}\right)$ where $g_{1}(z)=4(1+z)^{3} /\left[(1+z)^{2}+1\right]^{2}$ from (74). The horizontal and vertical axes are redshift $z$. Of interest is the curved unity (1) contour going from horizontal lower right to vertical upper left in the graph. 


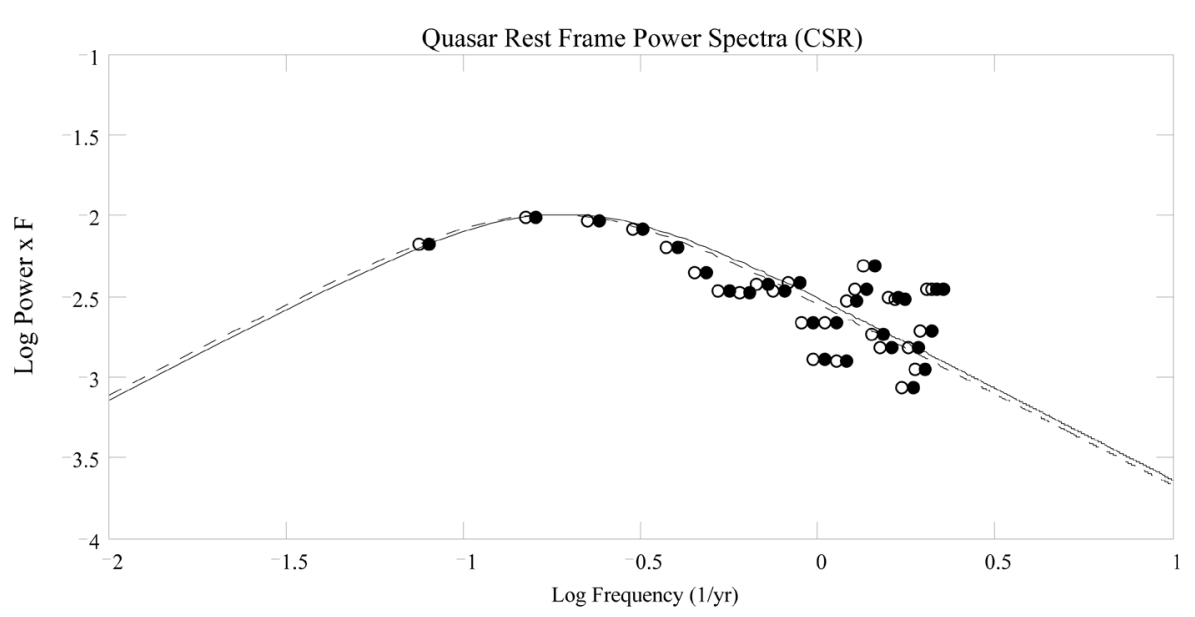

Figure 13. Simulated quasar light curve power spectra in the rest frame $K^{\prime}$ of each quasar, assuming each light curve transforms according to CSR. The frequency $f$ is $\mathrm{yr}^{-1}$. For the low redshift power spectrum, filled circles, $z_{1}=0.7$, the horizontal axis is scaled by $g_{1}\left(z_{1}\right)$ and the vertical axis has unit scaling. The power spectrum time interval is transformed by $1 / g_{1}\left(z_{1}\right)$ and the frequency is transformed by $g_{1}\left(z_{1}\right)$ which effectively makes the scale unity. For the high redshift power spectrum, open circles, $z_{2}=1.4$, the horizontal axis is scaled by $g_{1}\left(z_{2}\right)$ and the vertical axis has unit scaling although the power spectrum time interval is transformed by $1 / g_{1}\left(z_{2}\right)$ and the frequency is scaled by $g_{1}\left(z_{2}\right)$. The fitting function $P_{f}(f)$ has the same parameters as used at the origin of $K$ except the central frequency is scaled by $f_{c}(z)=0.14724 \times g_{1}(z)$ with $z=z_{1}$, solid line, and $z=z_{2}$, dashed line.

which is related [4] to the time $t_{r c}$ of recombination by $v_{r c} / c=t_{r c} / \tau$. Applying (58), we have

$$
\frac{v_{r c}}{c}=\frac{t_{r c}}{\tau}=\frac{\left(1+z_{r c}\right)^{2}-1}{\left(1+z_{r c}\right)^{2}+1},
$$

where $z_{r c}$ is the cosmological redshift at recombination. The coordinate distance $r_{b b}$ to the big bang is given by (23) with $v / c=1$,

$$
r_{b b}=\frac{c \tau}{\sqrt{1-\Omega_{M}}} \sinh \left(\sqrt{1-\Omega_{M}}\right) .
$$

Likewise, the coordinate distance $r_{r c}$ to the recombination epoch with $v / c=v_{r c} / c$, is given by

$$
r_{r c}=\frac{c \tau}{\sqrt{1-\Omega_{M}}} \sinh \left(\frac{v_{r c}}{c} \sqrt{1-\Omega_{M}}\right) .
$$

We construct a simple model to determine the size of the sound horizon [16] [17] for the longest sound wave, which generates the first acoustic peak. If $r_{e}$ is the radius of the sphere of expanding plasma, $\bar{v}_{e}=\left(c+v_{r c}\right) / 2$ is the average expansion velocity between the big bang and the recombination epoch and $c_{s}=c / \sqrt{3}$ is the speed of the longest sound wave in the plasma, then, by proportion of velocities, $r_{s h}=\left(c_{s} / \bar{v}_{e}\right) r_{e}$ is the radius of the sphere containing the longest wave. Assuming that the wave travels along a great circle path of the sphere, the size of the sound horizon $d_{s h}$ is given by

$$
d_{s h}=2 \pi r_{s h}=\frac{4 \pi r_{e}}{\sqrt{3}\left(1+v_{r c} / c\right)} .
$$

Defining $r_{e}=r_{b b}-r_{r c}$, which is the difference of (112) and (113), then from (114), the size of the sound horizon at recombination is given by 


$$
d_{s h}=\frac{4 \pi}{\sqrt{3}} \frac{\left(r_{b b}-r_{r c}\right)}{\left(1+v_{r c} / c\right)}=\frac{4 \pi c \tau}{\sqrt{3}}\left\{\frac{\sinh \left[\sqrt{1-\Omega_{M}}\right]-\sinh \left[\left(v_{r c} / c\right) \sqrt{1-\Omega_{M}}\right]}{\sqrt{1-\Omega_{M}}\left(1+v_{r c} / c\right)}\right\} .
$$

The angle $\theta_{s h}$ of the sound horizon at recombination is given by

$$
\theta_{s h}=\frac{d_{s h}}{D A_{r c}},
$$

where the angular diameter distance $D A_{r c}$ is given by [10]

$$
D A_{r c}=\frac{D L_{r c}}{\left(1+z_{r c}\right)^{2}},
$$

where $D L_{r c}$ is the luminosity distance of the recombination epoch. Substituting from (111)-(115) and (117) into (116) and simplifying we obtain

$$
\theta_{s h}=\frac{4 \pi}{\sqrt{3}}\left[\frac{\left(1+z_{r c}\right) \sqrt{1-t_{r c}^{2} / \tau^{2}}}{1+v_{r c} / c}\right]\left\{\frac{\sinh \left[\sqrt{1-\Omega_{M}}\right]}{\sinh \left[\left(v_{r c} / c\right) \sqrt{1-\Omega_{M}}\right]}-1\right\} .
$$

The CMB radiation escaped the matter sphere and expanded to fill all space. The size of the sound horizon $d_{s h 0}$ in the CMB on today's sky is obtained by applying (53) with $d_{s h}$,

$$
d_{s h 0}=d_{s h}\left(1+z_{r c}\right) .
$$

Then, the angle $\theta_{\text {sho }}$ of the sound horizon in the CMB radiation field on today's sky is given by

$$
\theta_{s h 0}=\frac{d_{s h 0}}{D A_{r c}}=\theta_{s h}\left(1+z_{r c}\right) \text {. }
$$

The multipole $l$ of the first acoustic peak [16] recorded in the CMB radiation field is proportional to the inverse of (120),

$$
l \approx \frac{\pi}{\theta_{s h 0}}=\frac{\pi}{\theta_{s h}\left(1+z_{r c}\right)} .
$$

Substituting $\Omega_{M}=0.800 \pm 0.080$ and $z_{r c}=1100$ into the above equations we obtain a value of

$$
l \approx 224 \pm 5 \text {, }
$$

which is in good agreement with observation [18] [19] and,

$$
\theta_{\text {sh } 0} \approx 0.805^{\circ} \pm 0.020^{\circ} \text {. }
$$

The size of the sound horizon on today's sky is $d_{\text {sh } 0} \approx 30.2 \mathrm{Mpc}$, which is $1 / 5$ the value of the standard model.

\section{Discussion}

Let us review briefly some aspects of the Carmeli five dimensional brane world cosmological model.

\subsection{Velocity, Acceleration and Cosmic Distances in CSR}

From (33), for $\mathrm{d} t=0$ with $\mathrm{d} x^{2}+\mathrm{d} y^{2}+\mathrm{d} z^{2}=\mathrm{d} r^{2}$ we have,

$$
\mathrm{d} s^{2}=\tau^{2} \mathrm{~d} v^{2}-\left(\mathrm{d} x^{2}+\mathrm{d} y^{2}+\mathrm{d} z^{2}\right)
$$

This can be manipulated to obtain

$$
1=\tau^{2}\left(\frac{\mathrm{d} v}{\mathrm{~d} s}\right)^{2}\left(1-\frac{\mathrm{d} x^{2}+\mathrm{d} y^{2}+\mathrm{d} z^{2}}{\tau^{2} \mathrm{~d} v^{2}}\right)=\tau^{2}\left(\frac{\mathrm{d} v}{\mathrm{~d} s}\right)^{2}\left(1-\frac{t^{2}}{\tau^{2}}\right)
$$

where 


$$
t^{2}=\left(\mathrm{d} x^{2}+\mathrm{d} y^{2}+\mathrm{d} z^{2}\right) / \mathrm{d} v^{2}
$$

is the cosmic time (squared). Using (125) this gives for the components of the four-velocity in CSR,

$$
u^{\mu}=\frac{\mathrm{d} x^{\mu}}{\mathrm{d} s}=\frac{\mathrm{d} x^{\mu}}{\mathrm{d} v} \frac{\mathrm{d} v}{\mathrm{~d} s}=\frac{1}{\tau \sqrt{1-t^{2} / \tau^{2}}} \frac{\mathrm{d} x^{\mu}}{\mathrm{d} v}=\frac{\gamma}{\tau} \frac{\mathrm{d} x^{\mu}}{\mathrm{d} v},
$$

where $\mu=0,1,2,3$ and

$$
\gamma=\frac{1}{\sqrt{1-t^{2} / \tau^{2}}}
$$

We have from (127) that

$$
\begin{gathered}
u^{0}=\gamma, \\
u^{k}=\frac{\gamma}{\tau} \frac{\mathrm{d} x^{k}}{\mathrm{~d} v}, k=\{1,2,3\} .
\end{gathered}
$$

Defining $u_{\mu}=u^{\mu}$ we obtain for the invariant 4-vector length, from (127), (129) and (130),

$$
u_{\mu} u^{\mu}=u_{0} u^{0}-u_{1} u^{1}-u_{2} u^{2}-u_{3} u^{3}=1,
$$

that is, the length of $u^{\mu}$ is unity in all CSR frames of reference. Multiplying (125) by $a_{0} \tau^{4}$, where $a_{0}$ is the ordinary acceleration measured in the cosmic frame at time 0 , the local frame, we obtain after some manipulation,

$$
a^{2} \tau^{4}-a^{2} t^{2} \tau^{2}=a_{0}^{2} \tau^{4}
$$

where

$$
a=\frac{a_{0}}{\sqrt{1-t^{2} / \tau^{2}}},
$$

is the acceleration at any cosmic time $t$. Equation (132) can be put into the form

$$
a^{2} \tau^{4}-\tau^{2} v^{2}=a_{0}^{2} \tau^{4}
$$

where

$$
v=a t=\frac{a_{0} t}{\sqrt{1-t^{2} / \tau^{2}}}
$$

is the velocity of a point which had an acceleration $a$ over a time $t$. Defining the cosmic distance $S=a \tau^{2}$ we have from (134)

$$
S^{2}-\tau^{2} v^{2}=S_{0}^{2},
$$

where $S_{0}=a_{0} \tau^{2}$. This is analogous to the energy equation in SR, $E^{2}-c^{2} p^{2}=E_{0}^{2}$. Refer to [20] for a thorough treatment of this topic.

\subsection{Behavior for Large Cosmic Time}

The cosmological redshift (57) and the cosmic aging function (74) are two functions which can be used to describe the behaviour expected at large cosmic time $t \approx \tau$, where $\tau$ is the Hubble-Carmeli time constant and is the largest possible time. For the cosmological redshift, for observations of events close to the big bang we have

$$
1+z \propto \frac{1}{\sqrt{1-t / \tau}} \rightarrow \infty \text { as } t \rightarrow \tau .
$$

The luminosity distance (78), as $t \rightarrow \tau, v / c \rightarrow 1$, has the form 


$$
D_{L}(t) \propto \frac{c \tau}{(1-t / \tau)} \rightarrow \infty \quad \text { as } t \rightarrow \tau .
$$

For the standard model, the luminosity distance relation, by (137), $D L_{S M} \propto c \tau / \sqrt{1-t / \tau}$, for large $t$. We see that the CGR luminosity distance, by (138), is larger than the standard model by the factor $1 / \sqrt{1-t / \tau}$. On the other hand, from the cosmic aging function (73), for an observation $\Delta t$ of an elapsed time $\Delta t^{\prime}$ which occurred close to the big bang time we have

$$
\Delta t=\Delta t^{\prime} g_{1}(t)=\Delta t^{\prime}(1+t / \tau) \sqrt{1-t^{2} / \tau^{2}} \rightarrow 0 \text { as } t \rightarrow \tau
$$

This implies that durations of events, such as for example star formation, star collapse or star bursts, observed in nearby galaxies at cosmic times $t<0.84 \tau$ (redshifts $z<3.4$ ) should be observed to have shorter durations the further back we look beyond cosmic times $t>0.84 \tau$ (redshifts $z>3.4$ ).

\subsection{The Cosmological Redshift vs, the Cosmic Aging Function}

One may ask why the cosmological redshift of the wave length $\lambda$ of light is given by the relation $(1+z) \lambda$ instead of with the cosmological aging function $g_{1}(t) \lambda$. The answer is that light wave phenomena do not involve the addition of cosmic times as do evolutionary phenomena such as a star burst or collapse. Light propagation is only affected by cosmic expansion while evolutionary phenomena are affected by cosmic time addition and cosmic expansion.

\subsection{The Accelerated Expansion}

CGR does not have a cosmological constant, but it does have a critical mass density $\rho_{c}$. From (14), the effective mass density can be defined in terms of a vacuum mass density

$$
\rho_{\text {eff }}=\rho+\rho_{\text {vac }}
$$

where

$$
\rho_{\text {vac }}=-\rho_{c}=-3 / 8 \pi G \tau^{2}
$$

is the constant negative mass density of the vacuum, which is not the common view of a vacuum density. Differentiating (18) with respect to $v$ we obtain the acceleration in space-velocity, which we put in the form

$$
\frac{\mathrm{d}^{2} r}{\mathrm{~d} v^{2}}+K r=0
$$

where

$$
K=\left(\rho+\rho_{\text {vac }}\right) / c^{2} \rho_{c}=(\Omega-1) / c^{2},
$$

and we have made the substitution, using (141),

$$
\Omega_{\text {vac }}=\rho_{\text {vac }} / \rho_{c}=-1 \text {. }
$$

Equation (142) is Hooke's law of the universe [7, Section 5.4] where $K$ is Hooke's constant for the universe. If $\Omega>1$ then $K$ is positive and its solution is a sum of sine and cosine functions and the universe has a decelerated expansion and is closed. If $\Omega<1$ then $K$ is negative and the solution is a sum of hyperbolic sinh and cosh functions, which means the universe has an accelerated expansion and is open; this is the situation in our universe today where we derived $\Omega=\Omega_{M}=0.800$. If $\Omega=1$ then $K=0$ and the universe is not accelerating and is neither open nor close.

Although beyond the scope of this paper, we give an expression [21] for the vacuum density $\rho_{\text {vac }}$ in relation to the Bekenstein-Hawking black hole entropy [22] given by $S=\left(k c^{3} A\right) /(4 \hbar G)$, where $k$ is Boltzmann's constant, $\hbar$ is Planck's constant over $2 \pi$ and $A=4 \pi c^{2} \tau^{2}$ is the area of the event horizon. For our universe of mass $M=\tau c^{3} / 4 G$, where the universe radius is twice the Schwarzschild radius, the entropy is given by 


$$
S=\frac{\pi k \tau^{2} c^{5}}{\hbar G}
$$

which can be put into the form relating to the vacuum mass density

$$
\rho_{\text {vac }}=\frac{-3}{8 \pi G \tau^{2}}=\frac{\rho_{P}}{(S / k)},
$$

where the cosmological Planck mass density $\rho_{P}=-M_{P} / L_{P}^{3}$. The cosmological Planck mass

$M_{P}=\sqrt{\sqrt{3 / 8} \hbar c / G}$ and length $L_{P}=\hbar / M_{P} c$. The value of $(S / k) \approx 1.980 \times 10^{122}$.

\subsection{Gravitational Waves as a Theoretic Selection Criteria}

When gravitational waves are detected we will be able to better quantify the strengths and weaknesses of the standard model (GR) and other models. In this regard, a paper on gravitational wave interferometry [23] has a good description of alternative theories to GR and is a fine starting place for further research. Along those lines of inquiry, [24] describes the behaviour of gravitational waves in Carmeli cosmology, predicting a highly attenuated result for gravitational waves from galactic sources but possible detectability for gravitational waves from within the Milky Way Galaxy.

\section{Conclusion}

In this paper, we used the linearized approximation of the 5-D Cosmological General Relativity as developed by Carmeli. A flat space CSR model was derived in a general way from the curved space CGR model. The CGR luminosity distance relation was applied to SCP Union 2.1 SNe-Ia distance data up to redshift $z<1.5$ combined with GRB distance data up to redshift $z<7$. Utilizing the reduced $\chi^{2}$ method in the data analysis, it was shown that the CGR model with a best fit mass density of $\Omega_{M}=0.800 \pm 0.080$ performed as well as the $\Lambda C D M$ flat space model with an apriori mass density of $\Omega_{M}=0.270$. Regarding the hypothetical $X$ particle constituents, the CGR model determines rest mass energies for the $\bar{Y}$ and $\Phi^{*}$ particles of $m_{\bar{Y}} c^{2}=m_{\Phi^{*}} c^{2} \approx 9.79 \pm 0.47 \mathrm{GeV}$. We also found that CSR could confirm the $\Lambda C D M$ model result of time dilation of $(1+z)$ in SNe-Ia light curves. We also showed how the cosmic aging function $g_{1}(z)$ produced a null effect of time dilation in simulated light curve power spectra between two groups of hypothetical QSO's separated by a redshift $\Delta z \approx 1.0$. Finally, we gave a model for obtaining the first acoustic peak of the CMB anisotropy, deriving a multipole $l \approx 224 \pm 5$, in good agreement with observation, and with an angle on the sky of $\theta_{\text {sh } 0} \approx 0.805^{\circ} \pm 0.020^{\circ}$.

\section{References}

[1] Hawkins, M.R.S. (2010) On Time Dilation in Quasar Light Curves. Monthly Notices of the Royal Astronomical Society, 405, 1940-1946. http://dx.doi.org/10.1111/j.1365-2966.2010.16581.x

[2] Goldhaber, G., Groom, D.E., Kim, A., Aldering, G., Astier, P., Conley, A., et al. (2001) Timescale Stretch Parameterization of Type Ia Supernova B-Band Light Curves. The Astrophysical Journal, 558, 359-368. http://arxiv.org/abs/astro-ph/0104382 http://dx.doi.org/10.1086/322460

[3] Blondin, S., Davis, T.M., Krisciunas, K., Schmidt, B.P., Sollerman, J., Wood-Vasey, W.M., et al. (2008) Time Dilation in Type Ia Supernova Spectra at High Redshift. The Astrophysical Journal, 682, 724-736. http://arxiv.org/abs/0804.3595 http://dx.doi.org/10.1086/589568

[4] Carmeli, M. (2002) Cosmological Special Relativity. Second Edition, World Scientific, Singapore.

[5] Supernova Cosmology Project. 26 January 2016. http://www.supernova.lbl.gov/Union/figures/SCPUnion2.1 mu vs z.txt

[6] Schaefer, B.E. (2007) The Hubble Diagram to Redshift $>6$ from 69 Gamma-Ray Bursts. The Astrophysical Journal, 660, 16-46. http://www.arxiv.org/abs/astro-ph/0612285 
http://dx.doi.org/10.1086/511742

[7] Carmeli, M. (2008) Relativity: Modern Large-Scale Spacetime Structure of the Cosmos. World Scientific, Singapore.

[8] Davoudiasl, H., Morrissey, D.E., Sigurdson, K. and Tulin, S. (2010) Unified Origin for Baryonic Visible Matter and Antibaryonic Dark Matter. Physical Review Letters, 105, Article ID: 211304. http://arxiv.org/abs/1008.2399 http://dx.doi.org/10.1103/physrevlett.105.211304

[9] Oliveira, F.J. and Hartnett, J.G. (2006) Carmeli’s Cosmology Fits Data for an Accelerating and Decelerating Universe without Dark Matter or Dark Energy. Foundations of Physics Letters, 19, 519-535. http://www.arxiv.org/abs/astro-ph/0603500v4 http://dx.doi.org/10.1007/s10702-006-1007-4

[10] Hartnett, J.G. and Oliveira, F.J. (2007) Luminosity Distance, Angular Size and Surface Brightness in Cosmological General Relativity. Foundations of Physics, 37, 446-454. http://www.arxiv.org/abs/astro-ph/0603500v5 http://dx.doi.org/10.1007/s10701-007-9108-X

[11] Greiner, J. (22 December 2015). http://www.mpe.mpg.de/ jcg/grbgen.html

[12] Weinberg, S. (2008) Cosmology. Oxford University Press, New York.

[13] Hartnett, J.G. (2008) Extending the Redshift-Distance Relation in Cosmological General Relativity to Higher Redshifts. Foundations of Physics, 38, 201-215. http://arxiv.org/abs/0705.3097 http://dx.doi.org/10.1007/s10701-007-9198-5

[14] Öztas, A.M. and Smith, M.L. (2014) The Cosmological Constant Constrained with Union2.1 Supernovae Type Ia Data. International Journal of Theoretical Physics, 53, 2636-2661. http://www.arxiv.org/abs/1401.0724 http://dx.doi.org/10.1007/s10773-014-2061-5

[15] Burles, S., Nollett, K.M. and Turner, M.S. (2001) Big-Bang Nucleosynthesis Predictions for Precision Cosmology. The Astrophysical Journal, 552, L1-L5. http://www.arxiv.org/abs/astro-ph/0010171

[16] Cornish, N.J. (2000) Using the Acoustic Peak to Measure Cosmological Parameters. Physical Review D, 63, Article ID: 027302. http://www.arxiv.org/abs/astro-ph/0005261

http://dx.doi.org/10.1103/physrevd.63.027302

[17] 11 January 2016. http://astro.uchicago.edu/ frieman/Courses/A411/Lecture8/Lecture8-2010.pdf

[18] Hinshaw, G., Weiland, J.L., Hill, R.S., Odegard, N., Larson, D., Bennett, C.L., et al. (2009) Five-Year Wilkinson Microwave Anisotropy Probe (WMAP) Observations: Data Processing, Sky Maps, and Basic Results. The Astrophysical Journal Supplement Series, 180, 225-245. http://www.arxiv.org/abs/0803.0732 http://dx.doi.org/10.1088/0067-0049/180/2/225

[19] Hanany, S., Ade, P., Balbi, A., Bock, J., Borrill, J., Boscaleri, A., et al. (2000) MAXIMA-1: A Measurement of the Cosmic Microwave Background Anisotropy on Angular Scales of 10'-5 . The Astrophysical Journal Letters, 545, L5L9. http://www.arxiv.org/abs/astro-ph/0005123

[20] Carmeli, M. (2001) Velocity, Acceleration and Cosmic Distances in Cosmological Special Relativity. http://www.arxiv.org/abs/astro-ph/0111260

[21] Oliveira, F.J. (2012) Particle Pair Production in Cosmological General Relativity. International Journal of Theoretical Physics, 51, 3993-4005. http://www.arxiv.org/abs/1203.4797 http://dx.doi.org/10.1007/s10773-012-1291-7

[22] Wikipedia: Black Hole Thermodynamics. http://www.wikipedia.org/wiki/Black_hole_thermodynamics

[23] Corda, C. (2009) Interferometric Detection of Gravitational Waves: the Definitive Test for General Relativity. International Journal of Modern Physics D, 18, 2275-2282. http://www.arxiv.org/abs/0905.2502 http://dx.doi.org/10.1142/S0218271809015904

[24] Hartnett, J.G. and Tobar, M.E. (2006) Properties of Gravitational Waves in Cosmological General Relativity. International Journal of Theoretical Physics, 45, 2181-2190. http://www.arxiv.org/abs/gr-qc/0603067 http://dx.doi.org/10.1007/s10773-006-9181-5 